\title{
Hybrid Search Heuristics to Schedule Bottleneck Facility in Manufacturing Systems
}

\author{
Ponnambalam S.G. ${ }^{1}$, Jawahar. $\mathrm{N}^{2}$ and Maheswaran. $\mathrm{R}^{3}$ \\ ${ }^{1}$ Monash University, \\ 2Thiagarajar College of Engineering, Madurai, \\ ${ }^{3} \mathrm{MEPCO}$ Schlenk Engineering College, Sivakasi \\ ${ }^{1}$ Malaysia, 2,3 India
}

\section{Introduction}

In a manufacturing system, whether it is a flow shop or job shop, often one of its facilities constrains the production flow and determines the production rate. It is the one that causes the bottleneck of the whole production environment. The bottleneck facility is seen as an input bay, where the orders get accumulated [Drobouchevitch, \& Strusevich, 2000, Drobouchevitch, \& Strusevich, 2001]. So, the scheduling of bottleneck facility problems is exceedingly important for several reasons, probably the most relevant of which is that good solutions to this problems provide a support to mange and model the behavior of more complex systems such as flexible manufacturing systems [Baker, 1995]. It is therefore an important problem from the application point of view. Scheduling bottleneck facility is the assignment of jobs to be processed on a bottleneck machine over time. The single machine problem addresses the bottleneck situation in scheduling literature. This chapter addresses $\varepsilon$ the problem characteristics, objectives, solution strategies and methodologies, and few o hybrid search heuristics for the bottleneck scheduling problems.

\section{Bottleneck Scheduling Problem}

\subsection{Problem Characteristics}

The bottleneck facility scheduling problem considered in this chapter is characterized by the following conditions:

- a set of $n$ independent jobs is available for processing at time zero and the job descriptors are known in advance

- a bottleneck facility is continuously available and is never kept idle

- the set up times for the jobs are independent of job sequence and can be included in processing times

- jobs are processed to completion without preemption

The various features of bottleneck machine are,

Jobs

ฐ Jobs are the activities that need to be scheduled on the bottleneck facility, where, only one $\widetilde{O}$ job can be processed at a time.

Source: Multiprocessor Scheduling: Theory and Applications, Book edited by Eugene Levner, ISBN 978-3-902613-02-8, pp.436, December 2007, Itech Education and Publishing, Vienna, Austria 


\section{Processing Time}

The processing time represents the period of time a job is actively assigned to the bottleneck facility. Usually, the assigned time is fixed and varies with each job.

\section{Preemption and Non-preemption}

Non-preemption disallows jobs from being interrupted by another job after processing has started. Most of the bottleneck scheduling problem considers non-preemption while there has been little research done with job preemption.

\section{Deadlines / Due Dates}

All jobs to be scheduled may have the same due dates and all the jobs must be processed before this date. But, in most real industry problems that has to deal with customer orders and product shipments, each job may possess different due dates [Tsiushuang et al., 1997]. The completion of a job after its due date is allowed, but a penalty is incurred. When the due date must absolutely be met, it is referred to as a deadline.

\section{Weight}

The weight of job is basically a priority factor. It denotes the importance of job relative to the other jobs in the system. For example, a weight may represent the actual cost of keeping the job in the system.

\subsection{Objectives}

With the well defined characteristics of scheduling problem, the motive of automated scheduling has been to significantly improve production line utilization and cost reduction. This may be achieved by imposing any of the following objective functions :

- Minimizing completion time, flow time and make span

- Minimizing the lateness

- Minimizing earliness and tardiness

- Minimizing weighted measures

- Multi-criteria objective

However, the current trends indicate that the minimization of total weighted tardiness objective is of much importance because of the following reasons. This is a crucial form of decision-making in manufacturing as well as in service industries. The buyer - vendor relationship plays an important role in business. Usually, buyers desire a reliable time delivery for meeting their schedules, and so the primary objective becomes to reduce the amount by which the individual completion times exceed the promised times, i.e. due dates. For example, when a company has to meet the shipping date on which it has committed its products to the customers and the production time depends to a great extend on one resource, as is often the case, it is faced with the bottleneck facility total weighted tardiness problems. Thus the problem of how jobs' due dates can be met such that the cost of jobs being late, as measured by the weighted tardiness, is minimized. The ability to cope efficiently with this kind of problems will boost the company's competitiveness.

\subsection{Problem Definition}

A set of jobs (indexed $1,2,3, \ldots j \ldots n$ ) is to be processed without interruption on a bottleneck facility that can process one job at a time. All jobs become available for processing at time zero. $J^{\text {th }} j o b$ has an integer processing time $p_{j}$, a due date $d_{j}$, and a positive weight $w_{j}$. A weighted tardiness penalty is incurred for each time unit of tardiness $T_{j}$ if job $j$ is completed after its due date $d_{j}$. The tardiness value $T_{j}$ is zero when the job is completed before the due 
date and other wise is $\left(C_{j}-d_{j}\right)$ where $C_{j}$ is the completion time of the job [Bahram Alidaee \& Ramakrishnan, 1996]. The problem can be formally stated to find a sequence $\sigma$ that minimizes

$$
\mathrm{Z}(\sigma)=\sum_{j=1}^{n} w_{j} T_{j}
$$

\subsection{Complexity of the problem}

While scheduling $n$ jobs in a bottleneck facility, there is a one-to-one correspondence between a sequence of these $n$ jobs and a permutation of the job indices. The total number of different solutions to the scheduling bottleneck facility problem is $n$ !. Bottleneck machine scheduling problems are proved as NP- hard [Lawler, 1977; Du \& Leung, 1990]. That is, the time the best possible algorithm will need to solve the problem increases in the worst case exponentially with the size of the problem.

\section{Solution Methodologies}

The task in bottleneck scheduling problems is to find a permutation of jobs that meets the problem's objective best. Some of the scheduling algorithms viz. enumerative and branch and bound techniques, Langarangian method, construction heuristics, heuristic search algorithms etc. reported in the literature to solve the problem are presented below,

\subsection{Enumerative and Branch and Bound Techniques}

A straightforward strategy is to solve the bottleneck facility scheduling problems by enumerating all possible solutions and then pick the best one. Yet, this may take considerable time as there are $n$ ! no. of different sequences available for $n$ jobs. Fortunately there exist more complex methods like branch - and - bound algorithms that allow discarding parts of the search space in which the optimal solution cannot be found.

Lawler and Wood (1966) proposed a branch - and - bound technique which is a backtracking type algorithm that searches through the space of partial solutions. Potts and Van Wassenhove (1985) addressed implicit enumerative algorithms for the total weighted tardiness problem and observed that the state-of-the-art branch and bound algorithm yields optimality, but they require considerable computer resources both in terms of computation time and memory requirements. Abdul-Razaq et al., (1990) performed a computational comparison of several state-of-the-art exact algorithms for the bottleneck facility total weighted tardiness problems. Szwarc and Mukhopadhyay (1997) and Della Croce et al. (1998) presented branch and bound procedures for total tardiness problem.

\subsection{Langrangian relaxation method}

Another popular solution technique is integer-programming problems based Lagrangian relaxation method. Here the integer constraint which is the main problem is to be removed or relaxed. Shapiro (1979) made a survey about Lagrangian relaxation, which has been used in discrete optimization for many decades. Potts and Van Wassenhove (1982) combined Lawlers' decomposition theorem with the approach of Schrage and Baker, 1978, to implement an efficient algorithm to solve instances up to 100 jobs. 


\subsection{Construction heuristics}

Often solutions for problems are needed very fast, as the problem is an element of a dynamic real world setting. This requirement can generally not be met by exact algorithms like branch and bound algorithm and Lagrangian relaxation method, especially when the problem is NP hard. Besides, not everyone is interested in the optimal solution. In many cases, it is preferable to find a sub-optimal, but good solution in a short time which can be obtained by constructive algorithms. Most of the researchers have reported that the above enumerative and Lagranginan algorithms are computationally expensive for larger problem size and tend for other techniques viz. construction heuristics and heuristic search algorithms. Constructive algorithms generate solutions from scratch by adding solution components to an initially empty solution until it is complete. A common approach is to generate a solution in a greedy manner, where a dispatching rule decides heuristically which job should be added next to the sequence of jobs that makes up the partial solution. Dispatching rules have been applied consistently to scheduling problems. They are procedures designed to provide good solutions to complex problems in real-time. The term dispatching rule, scheduling rule, sequencing rule or heuristic are often used synonymously.

Panwalker and Iskander (1977) named construction heuristics as scheduling rules and made a survey about different scheduling rules. Blackstone et al. (1982) called as dispatching rules and discussed the state of art of various dispatching rules in the manufacturing operations. Haupt (1989) termed the construction heuristics as priority rules and provides a survey of this type of priority rule based scheduling. Montazer and Van Wassenhove (1990) extensively studied and analysed these scheduling rule using simulation techniques for a flexible manufacturing system.

A distinction in dispatching rules can be made as static and dynamic rules. Static rules are just a function of the a priori known job data and dynamic dispatching rules, on the other hand, depend on the partial solution constructed so far. An example of a static rule is Earliest Due Date (EDD) and an example of a dynamic rule is Modified Due Date (MDD). A possibility to get still better performing dispatching policies is to combine simple rules like $E D D$ or $M D D$. After having pilot investigations on the different dispatching rules, a Backward heuristic dispatching rule is suggested for bottleneck facility total weighted tardines problems which is described as below [Maheswaran, 2004] :

\subsubsection{Backward Heuristics (BH).}

$B H$ is a dynamic dispatching rule. It is a greedy heuristic procedure, in which the sequential job assignment starts from the last position and proceed backward towards the first position. The assignments are complete when the first position is assigned a job. The process consists of the following steps:

Step 1: Note the position in the sequence in which the next job is to be assigned. The sequence is developed starting from position $n$ and continuing backward to position 1 . So, the initial value of the position counter is $n$.

Step 2: Calculate $T$, which is the sum of the processing times for all unscheduled jobs.

Step 3: Calculate the penalty for each unscheduled job $i$ as $\left(T-d_{i}\right) X w_{i}$. If $d_{i}>T$, the penalty is zero, because only tardiness penalties are considered. 
Step 4: The next job to be scheduled in the designated position is the one having the minimum penalty from step 3 . In the case of tie, choose the job with the largest processing time.

Step 5: Reduce the position counter by 1 .

Repeat steps 1 through 5 until all jobs are scheduled.

\section{Numerical Example:}

The backward heuristics is explained by a numerical example by considering a four jobs problem in which the processing time, due date and weight of the four jobs are given below,

$\begin{array}{cccc}\text { Job no. } & \text { Processing time } \boldsymbol{p}_{\boldsymbol{i}} & \text { Due Date } \boldsymbol{d}_{\boldsymbol{i}} & \text { Weight } w_{i} \\ 1 & 37 & 49 & 1 \\ 2 & 27 & 36 & 5 \\ 3 & 1 & 1 & 1 \\ 4 & 28 & 37 & 5\end{array}$

For backward heuristics, the sequence is developed from the fourth position and at this time $T=93$ and penalty for job 1 is 44 , job 2 is 285 , job 3 is 93 and job 4 is 280 . The job 1 is having the minimum penalty and scheduled at the fourth position of the sequence.

For the third position, $T=56$ and penalty for the job 2 is 100, job 3 is 55 and job 4 is 140 . Now, job 3 is having minimum penalty and scheduled at the third position of the sequence. For, the second position, $T=55$ and the penalty of job 2 is 95 and job 4 is 90 and so job 4 is scheduled ant second position and job 2 is scheduled at first position of the sequence.

The resultant sequence generated from the backward phase is $2-4-3-1$ with a total weighted tardiness value of 189 .

\subsection{Heuristic Search Algorithms}

Heuristic search algorithms are often developed and used to solve many difficult NP-hard type computational problems in science and engineering. Since uninformed search by enumeration methods seems computational prohibitive for large search spaces, heuristic search receives increasing attention [Morton \& Pentico, 1993]. Heuristics can derive near optimal solutions in considerably less time than the exact algorithms. Heuristics often seek to exploit special structures in a problem to generate good solutions quickly. However, there is no guarantee that heuristics will find an optimal solution.

Heuristics are obtained by

- using a certain amount of repeated trials,

- employing one or more agents viz. neurons, particles, chromosomes, ants, and so on,

- operating with a mechanism of competition and cooperation,

- embedding procedures of self modification of the heuristic parameters or of the problem representation.

Heuristic search algorithms utilize the strengths of individual heuristics and offer a guided way for using various heuristics in solving a difficult computational problem. According to Osman (1996), a heuristic search "is an iterative generation process which guides a subordinate heuristic by combining intelligently different concepts for exploring and exploiting the search spaces..." [Osman, 1996, Osman \& Kelly, 1996]. Heuristic search algorithms have shown promise for solving "...complex combinatorial problems for which optimization methods have failed to be effective and efficient." 
A wide range of different heuristic search techniques have been proposed. They have some basic component parts in common and are:

- A representation of partial and complete solutions is required.

- Operators, which either extend partial solutions or modify complete solutions are needed.

- An objective function, which either estimates the costs of partial solutions or determines the costs of complete solutions is needed.

- The most crucial component of heuristic search techniques is the control structure that guides the search.

- Finally, a condition for terminating the iterative search process is required.

Common heuristic methods include:

- $\quad$ Tabu search, [Glover 1989; 1990; Glover et al., 1993; 1995],

- $\quad$ simulated annealing [Kirkpatrick et al., 1983],

- greedy random adaptive search procedures (GRASP) [Deshpande \& Triantaphyllou, 1998; Feo \& Resende, 1995],

- $\quad$ iterated local search [Helena et al., 2001],

- genetic algorithms [Goldberg, 1989], and

- $\quad$ ant colony optimization [Den Besten et al., 2000].

Instead of searching the problem space exhaustively, Reeves (1993) informs that modern heuristic techniques concentrate on guiding the search towards promising regions of the search space. Prominent heuristic search techniques are, among others, simulated annealing, Tabu search and evolutionary algorithms. The first two of them have been developed and tested extensively in combinatorial optimization. To the contrary, evolutionary algorithms have their origin in continuous optimization. Nevertheless, the components of evolutionary algorithms have their counterparts to other heuristic search techniques. A solution is called an individual which is modified by operators like crossover and mutation. The objective function corresponds to the fitness evaluation. The control structure has its counterpart in the selection scheme of evolutionary algorithms.In evolutionary algorithms, the search is loosely guided by a multi-set of solutions called a population, which is maintained in parallel. After a number of iterations (generations) the search is terminated by means of some criterion.

\subsubsection{Classification of Heuristic Search Algorithms}

Depending upon the characteristics to differentiate between search algorithms, several classifications are possible and each of them being the results of a specific view point. The most important methods of classification are:

- Nature inspired vs Non nature inspired

- Population based vs Single point search

- Dynamic vs Static objective function

- One vs Various neighborhood structure

- Memory Usage vs Memory less method

Nature inspired vs Non nature inspired

Perhaps, the most intuitive way of classifying heuristic search algorithms is based on the origin of the algorithms. There are nature inspired algorithms like evolutionary algorithms and ant algorithms, and non nature inspired algorithms like Tabu search and iterated local search / improvement algorithms. This classification is not meaningful for the following 
two reasons. First, many hybrid algorithms do not fit in either class or in a sense that it fit both at the same time. Second, sometimes it is difficult to clearly tell the genesis of an algorithm.

\section{Population based vs Single point search}

Another characteristic which can be used for the classifications is the way of performing the search. Does the algorithm work on a population or on a single solution at a time? Algorithms working on single solution are called as trajectory methods and encompass local search based heuristics. They all share the property of describing a trajectory in the search space during the search process. Population based methods on the contrary perform search process which describe the evolution of a set of points in the solution space.

\section{Dynamic vs Static objective function}

Search algorithms can also be classified according to the way they make use of the objective function. While some algorithms keep the objective function given in the problem representation "as it is" and some others like guided local search will modify during the search. The idea behind this search is to escape from the local optima by modifying the search landscape. Accordingly, during the search the objective function is altered by trying to incorporate information collected during the search process.

\section{One vs Various neighborhood structure}

Most search algorithms work on single neighborhood structure. In other words, the fitness landscape, which is searched doesn't change in the course of the algorithm. Other algorithms use a set of neighborhood structures which gives the possibility to diversify the search and tackle the problem jumping between different landscapes

\section{Memory Usage vs Memory less method}

A very important feature to classify the heuristic search algorithms is whether they use memory of search history or not. Memories less algorithms perform a Markov process, as the information they need is only the current state of the search process. There are several different ways of making use of memory. Usually it will be differentiated between short term and long term memory structures. The first usually keeps track of recently performed moves, visited solutions or, in general, decisions taken. The second is usually the accumulation of synthetic parameters and indexes about the search. The use of memory is nowadays recognized as one of the fundamental elements of the powerful heuristics.

\section{Hybrid Algorithms Developed}

The main objective of this work is to formulate different hybrid search heuristics which are designed to solve the problems of higher sizes within reasonable time. In this work, three different heuristic search algorithms are formulated and used to solve the bottleneck scheduling problems with objective of minimizing the total weighted tardiness.

They are:

- Heuristic Improvement algorithm [Maheswaran \& Ponnambalam, 2003]

- Iterated Local Improvement Evolutionary Algorithm [Maheswaran \& Ponnambalam, 2005]

- $\quad$ Self Improving Mutation Evolutionary Algorithms [Maheswaran et al., 2005] 


\subsection{Heuristic Improvement algorithm (HIA)}

Heuristic Improvement algorithm is devised in such a way to improve an initial sequence generated by construction heuristics. Generally, construction heuristics can be used to get the solution to the scheduling problems in a faster way. Construction heuristics generate solutions from scratch by adding solution components to an initially empty solution until it is complete. But, the results of these heuristics are not accurate. A common approach is to generate a solution in a greedy manner, where a dispatching rule decides heuristically which job should be added next to the sequence of jobs that makes up the partial solution. After pilot anlaysis, it is observed that the dynamic backward dispatching rules based on heuristics is performing well. It is proposed to apply a greedy heuristic improvement algorithm, which will operate on the sequence developed by backward heuristic as initial sequence for the improvement.

\subsubsection{Procedural Steps of Heuristic Improvement Algorithm}

The proposed heuristic improvement algorithm adopts the forward heuristic method addressed by Sule (1997) operating on some initial sequence. The procedure is out lined below:

Step 1: Initialize the sequence with backward heuristics and set its total weighted tardiness value as the objective value. The sequence obtained from backward heuristic is assumed to be the initial sequence and this is the best sequence at this stage with the total weighted tardiness as the objective value.

Step 2: Let $k$ define the lag between two jobs in the sequence that are exchanged. For example, jobs occupying positions 1 and 3 have a lag $k=2$.

Step 3: Perform the forward pass on the job sequence found in the backward phase that is the best sequence at this stage. The forward pass progresses from the job position 1 towards the job position $n$.

Step 3.1: Set $\mathrm{k}=\mathrm{n}-1$

Step 3.2: Set exchange position $j=k+1$

Step 3.3: Determine the savings by exchanging two jobs in the best sequence with a lag of $k$. The job scheduled in position $j$ is exchanged with the job scheduled in a position $(j-k)$. If $(j-k)$ is zero or negative then go to step 3. 6 . Calculate the penalty after exchange and compare it to the best sequence penalty.

Step 3.4:If there is either positive or zero savings in step 3.3, then go to step 3.5; otherwise the exchange is rejected. Increase the value of $j$ by one. If $j$ is equal to or less than $n$, then go to step 3.3. If $j>n$, then go to step 3.6.

Step 3.5: If the total penalty has decreased, the exchange is acceptable. Perform the exchange. The new sequence is now the best sequence; Go to step 3.1. Even if the savings is zero, make the exchange and go to step 3.1, unless the set of the jobs associated in this exchange has been checked and exchanged in an earlier application of the forward phase. In that case, no exchange is made at this time. Increase the value of $j$ by one. If $j<n$, then go to step 3.3. If $j=n$, then go to step 3.6.

Step 3.6: Decrease value of $k$ by one. If $k>0$, then go to step 2. If $k=0$, then go to step 4.

Step 4: The resulting sequence is the best sequence generated by this procedure. 


\section{Numerical Example :}

The four jobs problem given in section 3.3.1 is further improved by the forward phase. The sequence generated by backward phase $2-4-3-1$ with a total weighted tardiness value of 189 is consider as the best sequence at this stage. Set Lag $k=n-1$ which yields $k=3$. Exchange jobs in the position between $j \&(j+k)$. So, in the present sequence exchange job 2 and job 1 and the new sequence is $1-4-3-2$ which yields a total weighted tardiness value of 420 and there is no savings and the exchange is not accepted.

There is no more exchange possible for the lag $k=3$ and reduce $k$ by one which yields $k=3$.

Exchange job 2 and job 3, which yields the sequence 3-4-2-1 with value 144 . As there is savings and accept the change and this is the best sequence now.

Once again set the lag $k=3$, and repeat the procedure for the new sequence and finally the optimum sequence will be $3-2-4-1$ with a total weigted tardiness of 139 .

The forward phase algorithm is described by means of a flowchart as shown in the figure 1.

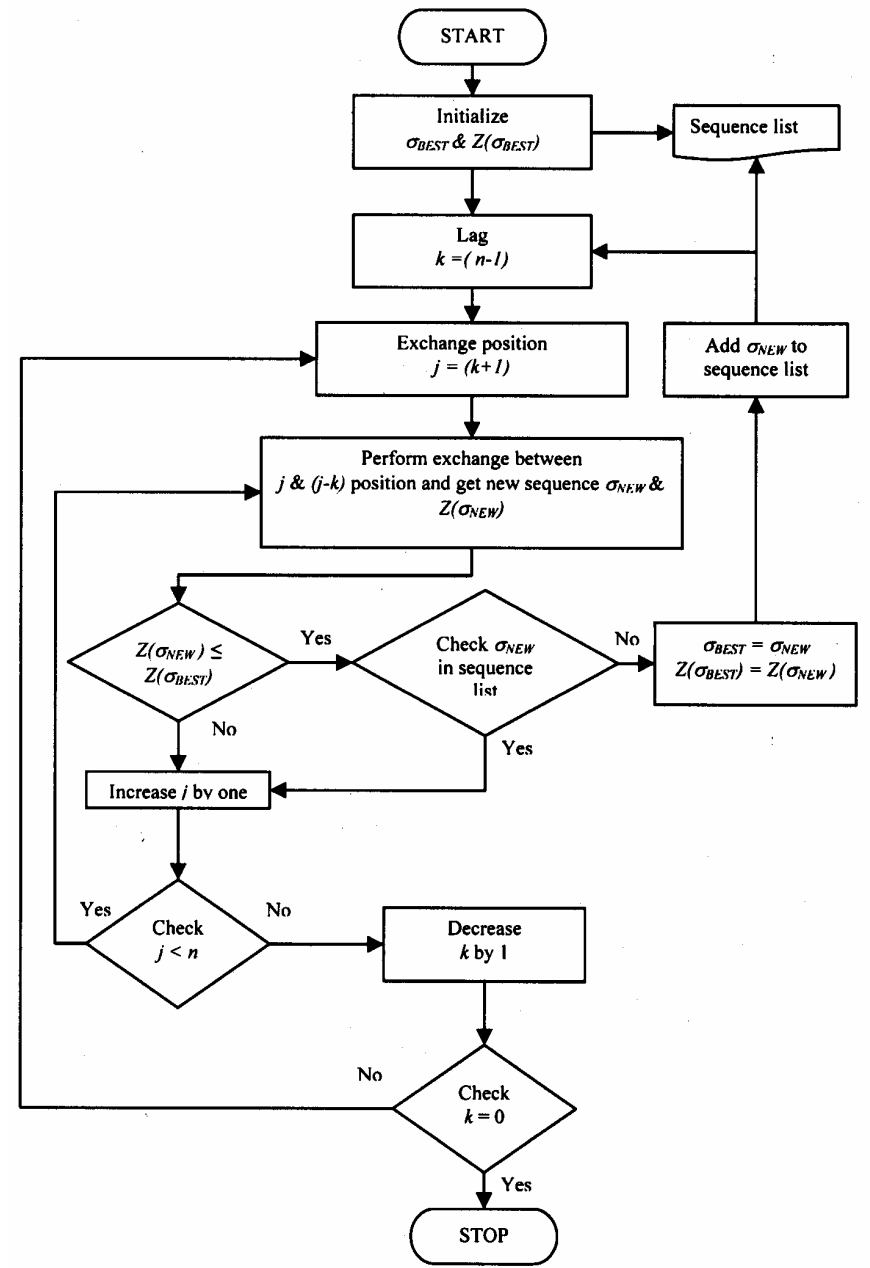

Figure 1. Heuristic Improvement Algorithm 


\subsection{Iterated Local Improvement Evolutionary Algorithm (ILIEA)}

According to the survey of Thomas Baeck et al. (1991), on the Evolution Strategies and its community has always placed more emphasis on mutation than crossover. The role of local search in the context of evolutionary algorithms and the wider field of evolutionary computing has been much discussed. In its most extreme form, this view casts mutation and other local operators as mere adjuncts to recombination, playing auxiliary (if important) roles such as keeping the gene pool well stocked and helping to tune final solutions. Radcliffe and Surry. (1994) investigated that a greater role for mutation, hill-climbing and local refinements are needed for evolutionary algorithms. Ackley (1987) recommends genetic hill climbing, in which crossover plays a rather less dominant role.

Iterated local improvement evolutionary algorithm is designed similar to an iterated local improvement algorithm with evolutionary based perturbation tool. Iterated local improvement algorithm is a simple but effective procedure to explore multiple local minima, which can be implemented in any type of local search algorithm. It is to perform multiple runs with the algorithm and each using a different starting solution. A promising but relatively unexplored idea is to restart near a local optimum, rather than from a randomly generated solution. Under this approach, the next starting solution is obtained from the current local optimum where the current local optimum is usually either the best local optimum found so far from the history, or the most recently generated local optimum by applying a pre-specified type of random move to it which is referred as kick or perturbation.

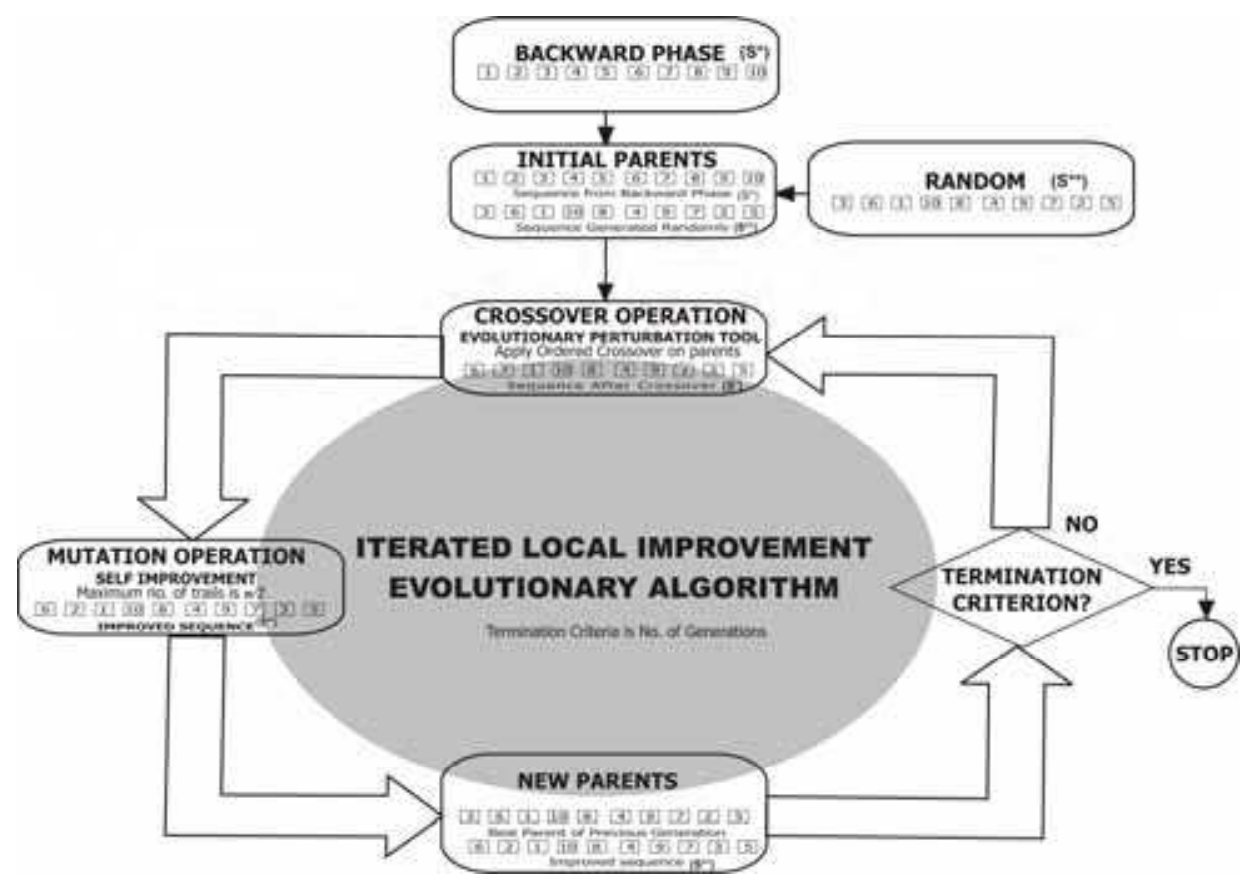

Figure 2. Iterated Local Improvement Evolutionary Algorithm 
Iterated Local Improvement Evolutionary Algorithm (ILIEA) is hybrid algorithm having $P O P=2$. The complexity of the algorithm is governed by the number of iterations used for termination criterion. The complete process of iterated local improvement evolutionary algorithm with an example is given in the figure 2. It consists of the following modules:

- Initial parents generation

- $\quad$ Population size $P O P=2$

- Crossover operation (Evolutionary perturbation technique)

- Crossover probability $\left(P_{c}\right)=1$

- Mutation operation (Self improvement technique)

- Mutation probability $\left(P_{m}\right)=1$

- New parents generation

\subsubsection{Initial Parents Generation}

A sequence of the bottleneck facility scheduling problem is mapped into a chromosome with the alleles assuming different and non repeating integer values in the $[1, n]$ interval. Any sequence can be mapped into this permutation representation. This approach can be found in most genetic algorithm articles dealing with sequencing problems [Franca et al., 2001]. The total weighted tardiness of a sequence is assumed to be the fitness function for ILIEA.

In this algorithm the population size is assumed to be two and the sequence developed by the backward phase acts as one parent and sequence generated taking events in a random order acts as the other parent.

\subsubsection{Crossover Operation (Evolutionary Perturbation Technique)}

Perturbation is a pre-specified type of random move applied to a solution. For a current solution $s^{*}$, a change or perturbation is applied to an intermediate state $s^{\prime}$. Then the Local Improvement is applied on $s^{\prime}$ and a new solution $s^{* \prime}$ is reached. If $s^{* \prime}$ passes an acceptance test, it becomes the next base solution for the search otherwise it returns to $s^{*}$. The overall procedure is shown in figure 3.

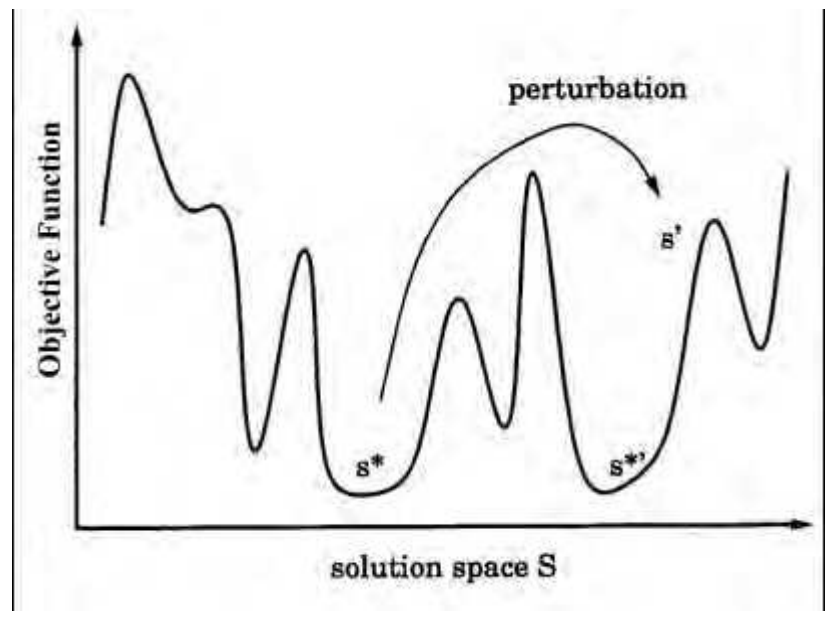

Figure 3. Procedures for Perturbation 
The crossover operation adopted in this work uses an evolutionary perturbation technique, which involves the following processes:

- Iterated local search (ILS)

- Perturbation tool

- Perturbation strength

- Acceptance criterion

Iterated Local Search: The underlying idea of ILS is that of building a random walk in $S^{*}$, the space of local optima defined by the output of a given local search. Four basic ingredients are needed to derive an ILS:

- a procedure to GenerateInitialSolution, which returns some initial solution,

- a local search procedure for LocalSearch,

- a scheme of how to perturb a solution, implemented by a procedure Perturbation, and

- an AcceptanceCriterion, which decides from which solution the search is continued.

The particular walk in $S^{*}$ followed by the ILS can also depend on the search history, which is indicated by history in Perturbation and AcceptanceCriterion.

The effectiveness of the walk in $S^{*}$ depend on the definition of the four component procedures of ILS: The effectiveness of the local search is of major importance, because it strongly influences the final solution quality of ILS and its overall computation time. The perturbations should allow the ILS to effectively escape local optima but at the same time avoid the disadvantages of random restart. The acceptance criterion, together with the perturbation, strongly influence the type of walk in $S^{*}$ and can be used to control the balance between intensification and diversification of the search. The initial solution will be important in the initial part of the search. The configuration problem in ILS is to find a best possible choice for the four components such that best overall performance is achieved. The algorithm outline of iterated local search is given in the figure 4 .

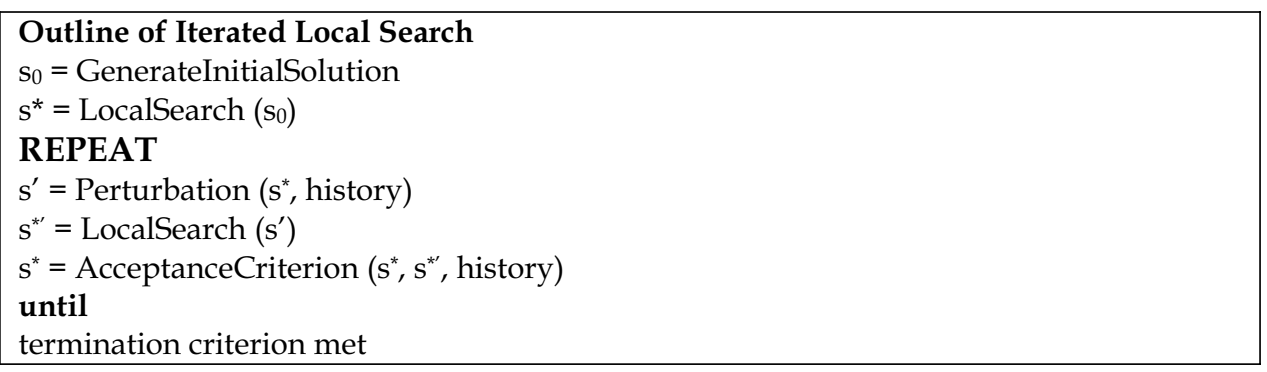

Figure 4. Iterated Local Search

Perturbation Tool :Though many researchers followed different types of perturbation tools, an evolutionary operator perturbation tool is used in this work. Here, an ordered crossover operator $(O X)$ is used as perturbation tool. The operation of the $O X$ is given as follows: The operator takes the initial sequence $s^{*}$ from the base heuristics and another sequence $s^{* *}$ is generated randomly. The resultant sequence $s^{\prime}$ will take, a fragment of the sequence from $s^{*}$ and the selection of the fragment is made uniformly at random. In the second phase, the empty positions of $s^{\prime}$ are sequentially filled according $s^{* *}$. The accepted $s^{*}$ for the next iteration will replace with worst of the previous $s^{*}$ and $s^{* *}$.

As an example, the sequence $s^{\prime}$ inherits the elements between the two crossover points, inclusive, from $s^{*}$ in the same order and position as they appeared. The length of the 
crossover is in the range between a random number generated in the range of $[1, n-1]$ job position as lower limit $(L L)$ and a random number generated in the range of $[L L, n]$ as the upper limit $(U L)$. The remaining elements are inherited from the alternate sequence $s^{* *}$ in the order in which they appear, beginning with the first position following the second crossover point and skipping over all elements already present in $s^{\prime}$.

An example for the perturbation tool is given in figure 5 . The elements $\alpha, \Gamma, v, \delta$ and $\omega$ are inherited from $s^{*}$ in the same order and position in which they occur. Then, starting from the first position after the second crossover point, $s^{\prime}$ inherits from $s^{* *}$. In this example, position 8 the next position, $s^{\prime}[8]=0$, which is already present in the offspring, so $s^{* *}$ is searched until an element is found which is not already present in $s^{\prime}$. Since $v, \omega$ and $\Gamma$ are already present in $s^{\prime}$, the search continues from the beginning of the string and $s^{\prime}[8]=s^{* *}[2]$ $=\beta, s^{\prime}[9]=s^{* *}[3]=\gamma, s^{\prime}[10]=s^{* *}[5]=\varepsilon$, and so on until the new sequence is generated [Starkweather. T. et al., 1991].

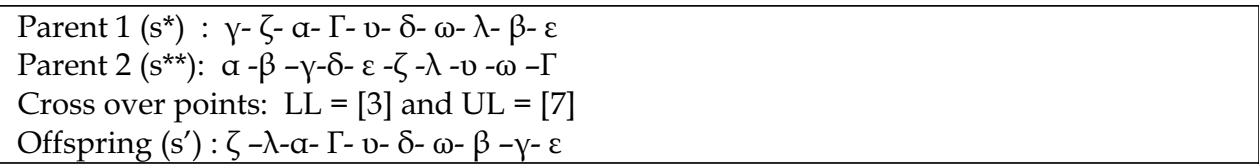

Figure 5. Ordered Crossover (OX)

Perturbation Strength: For some problems, appropriate perturbation strength is very small and seems to be rather independent of the instance size. The strength of a perturbation is referred as the number of solution components directly affected by a perturbation. The $O X$ operator will change most of the solution components in the sequence according to the generated $L L \& U L$ values.

Acceptance Criteria : The perturbation mechanism together with the local improvement defines the possible transitions between a current solution $s^{*}$ to a "neighboring" solution $s^{*}$. The acceptance criteria determines whether $s^{* \prime}$ is accepted or not as the new current solution. A natural choice for the acceptance criterion is to accept only better solutions which are a very strong intensification for search. This is termed as BETTER criterion. Diversification of the search is extremely favored if every $s^{* \prime}$ is accepted as the new solution. This is termed as random walk $(R W)$ criterion which is represented as

$$
\operatorname{RW}\left(\mathrm{s}^{*}, \mathrm{~s}^{*^{\prime}}, \text { history }\right):=\mathrm{s}^{*^{\prime}}
$$

Since, the operator $O X$ completely changes most of the solution components, the acceptance criterion is chosen as $R W$.

The sequence obtained after perturbation is further improved in the mutation operation which is self improving.

\subsubsection{Mutation Operation (Self Improvement Technique)}

The mutation operation adopted in this research uses a self improvement technique, which consists of the following parts:

- Local search

- Neighborhood structure

Local Search : Local search methods move iteratively through the solution set $S$. Based on the current and may be on the previous visited solutions, a new solution is chosen. The choice of the new solution is restricted to solutions that are somehow close to the current 
solution i.e. in the 'neighborhood' of the current solution. Different local search methods may be formulated depending on the method of choosing solutions from the neighborhood of the current solution and the way in which the stopping criteria are defined [Helena, 1995]. A neighborhood search method requires a representation of solutions to be chosen, and an initial solution to be constructed by some heuristic rule or created randomly. A neighbor is generated by some suitable mechanism, and an acceptance rule is used to decide whether it should replace the current solution or not. The acceptance rule in a neighborhood search method usually requires the comparison of objective function values for the current solution and its neighbor.

Neighborhoods are usually defined by first choosing a simple type of transition to obtain a new solution from a given one, and then defining the neighborhood as the set of all solutions that can be obtained from a given solution by performing one transition. Generally, a local search method is based on the following two routines:

- Given an instance, construct an initial solution.

- Given an instance and any solution, determine if there is a neighboring solution of lower cost, and if so, return one such solution. If no such solution exists, then the input solution is returned and it is indicated that it is a local optimal solution.

The basic structure of a local search is presented in figure 6

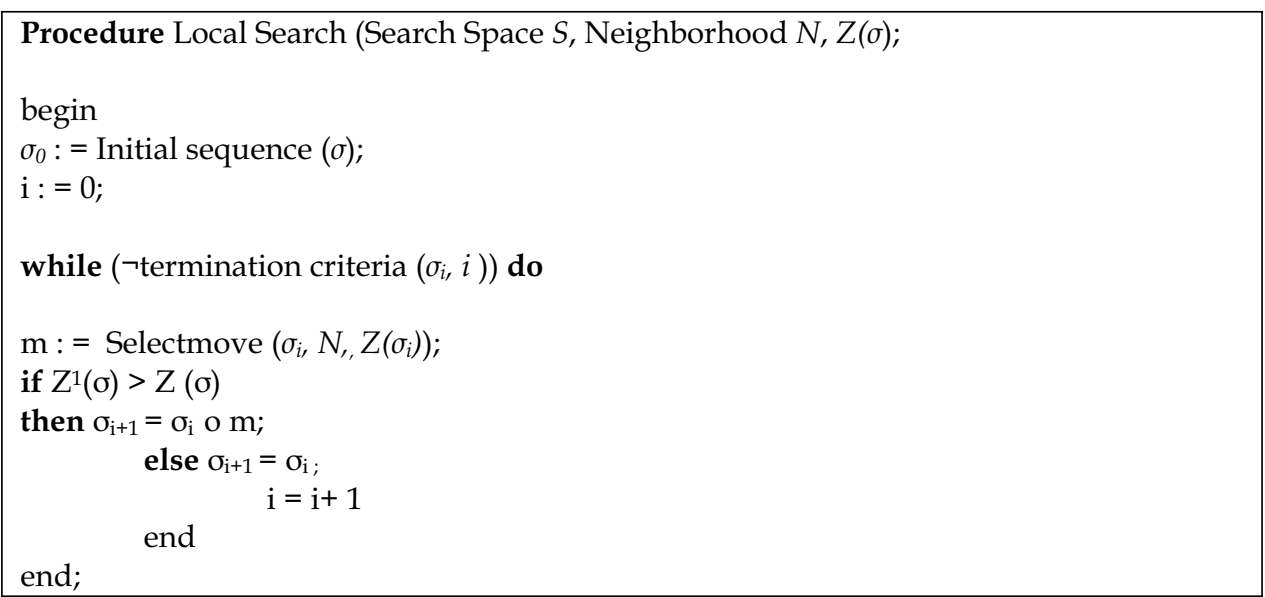

Figure 6. Local Search

Neighborhood Structure : Before applying local search methods to any problem a neighborhood structure is to be defined. A systematic way of defining neighborhoods is needed; otherwise, it is not possible to store the neighborhood. The neighborhoods define a frame for the possibilities of walking through the solution space; they have a crucial influence on the behavior of local search. If neighborhoods are small, the walk is very restricted and, thus, it may be hard to reach good solutions. On the other hand, if neighborhoods are large, it may be time consuming to decide in which direction (i.e. to which neighbor) the search shall continue. However, not only the size but the more the quality of the solutions in a neighborhood is of interest. If a neighborhood contains promising solutions, it does not matter if the size of the neighborhood is small and, on the other hand, large neighborhoods with only solutions of poor quality are not very helpful. 
Three common neighborhood schemes are used for scheduling problems and are given below:

- Adjacent neighborhood interchange in which a job may be swapped with jobs directly to its left or right in the schedule.

- Swap in which any two jobs in the schedule can be swapped.

- Insert in which a job is taken from its current position and placed in another position in the schedule.

In this work, four mechanisms are used for finding the neighborhood solutions to solve the bottleneck facility scheduling problems are investigated. They are:

- Adjacent neighborhood interchange

- Randomized neighborhood structure

- Randomized adjacent interchange $\left(\psi_{a i}\right)$,

- Randomized sliding mutation $\left(\psi_{s l}\right)$ and

- Randomized pair wise interchange $\left(\psi_{p w}\right)$

Adjacent neighborhood interchange

The process of the adjacent neighborhood interchange mechanism is shown in figure 7. For any solution $s$, neighbourhood of $s, N(s)$, includes $(n-1)$ different alternative neighbouring solutions obtained by interchanging a job with its right job in the sequence.

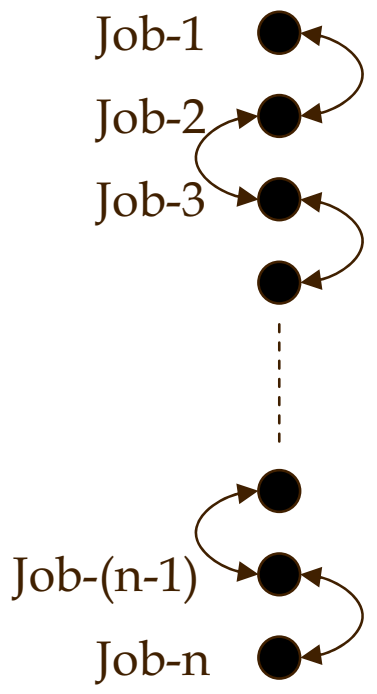

Figure 7. Adjacent Neighborhood Interchange

Randomized Adjacent Interchange $\left(\boldsymbol{\Psi}_{a i}\right)$

This is a randomized version of adjacent interchange neighborhood structure. This operator will generate a random number $(R)$ in the range $[1, n]$ and just interchanges the job present in the position $R$ with the next job in the sequence $(R+1)$ and represented as:

$$
\Psi_{\text {ai }}(\text { vпіj } \omega)=\text { vпji } \omega
$$




\section{Randomized Sliding Mutation $\left(\boldsymbol{\psi}_{s}\right)$}

This is a randomized version of inert neighborhood structure. This operator may be also termed as randomized extraction and backward shift insertion operator. Sliding mutation refers to "moving a job from the $j^{\text {th }}$ place and placing it before the $i^{\text {th }}$ position". Two values are generated randomly $\left(R_{1}\right.$ and $\left.R_{2}\right)$ in the range $[1, n]$ in such a way that $R_{1}<R_{2}$ and applied to jobs present in the positions in between $R_{1}$ and $R_{2}$. The job in position $R_{2}$ is placed before the job in position $R_{1}$ and all jobs in between $R_{1}$ and $R_{2}$ are pushed one position and represented as:

$$
\psi_{\text {sl }}(\text { viпj } \omega)=\text { vjiп } \omega
$$

Randomized Pair wise Interchange $\left(\boldsymbol{\Psi}_{p w}\right)$

This operator may be also termed as random swap operator and similar to swap neighborhood structure. Random swap refers to "the swapping according to the randomly generated values". Two values are generated randomly $\left(R_{1}\right.$ and $\left.R_{2}\right)$ in the range $[1, n]$ and applied to jobs present in the positions $R_{1}$ and $R_{2}$ and the jobs are swapped according to the random values generated and represented as:

$$
\Psi_{\text {pw }}(\text { vіпј } \omega)=\text { vјпi } \omega
$$

The improvement technique will be stopped with a maximum number of trials which is assumed to be a function related to number of jobs $(n)$.

The local search with different neighborhood structures with a termination criteria $n^{*} n^{*} n$ number of iterations, so that the complexity of the algorithm is in the order of $O\left(n^{3}\right)$, applied on the initial sequence obtained by backward phase heuristics.

The potentials of three randomized neighborhood structure are investigated by applying on the sequences generated by the $E D D, M D D$ and $B H$ heuristics as initial sequences. These local search is applied for a termination criteria $n^{*} n^{*} n$ number of iterations so that the complexity of the algorithm is in the order of $O\left(n^{3}\right)$. It is observed that the local search algorithm with adjacent neighborhood interchange is applied on the sequence generated by backward heuristics is not able to improve further and it is decided to use the randomized neighborhood structure. For large sizes of $n, \psi_{p w}$ structure can be applied as self improving technique in this proposed iterated local improvement evolutionary algorithm with a maximum number of trials for local improvement, which can be assumed as a function of size of the problem.

\subsubsection{New Parent Generation}

In this proposed algorithm, the locally improved offspring obtained after self improvement technique is used as a parent for the next generation. Even though, the improved offspring value is less than the previous parents, it must be considered for the next generation. The best parent of the previous generation will act as the other parent and the evolution process is continued for the predetermined number of generation.

\subsection{Self Improving Mutation Evolutionary Algorithms (SIMEA)}

Evolutionary algorithms are generally used to solve problems of higher search spaces. The search space in bottleneck facility scheduling problems is quite large ( $n !)$. Evolutionary Algorithms (EA) is the term used to describe search methods based on the mechanics of natural selection and evolution. Evolutionary Algorithms are often presented as general 
purpose search methods. The evolutionary process can be simulated on a computer in a number of ways and two self improving mutation based evolutionary algorithms are designed in this work to improve the results obtained from iterated local improvement algorithm. Self Improving Mutation Evolutionary Algorithms (SIMEA) are population based evolutionary algorithms in which each individual represents a sequence and the population evolves through tournament selection, ordered crossover and self improving mutation. The selection of initial population and termination criteria plays a vital role in the quality of the solution and complexity of the algorithm. The process of self improving mutation evolutionary algorithm is explained as below,

Self Improving Mutation Evolutionary Algorithm (SIMEA) is a hybrid algorithm having population size $P O P=n$, Crossover probability $\left(P_{c}\right)=1$ and Mutation probability $\left(P_{m}\right)=1$. The complexity of the algorithm is governed by different parameters like size of the population $(P O P)$ used for evolution, maximum trials for self improving mutation $(M)$ and number of generation needed for termination. The complete process of self improving mutation evolutionary algorithm with an example is given in the figure 8 . It consists of the following parts:

- $\quad$ Sequence representation

- Initial population

- Selection Operator

- Crossover operator

- Self improving mutation operator

- Termination criterion

The proposed self improving mutation evolutionary algorithm is shown in the figure 8 .

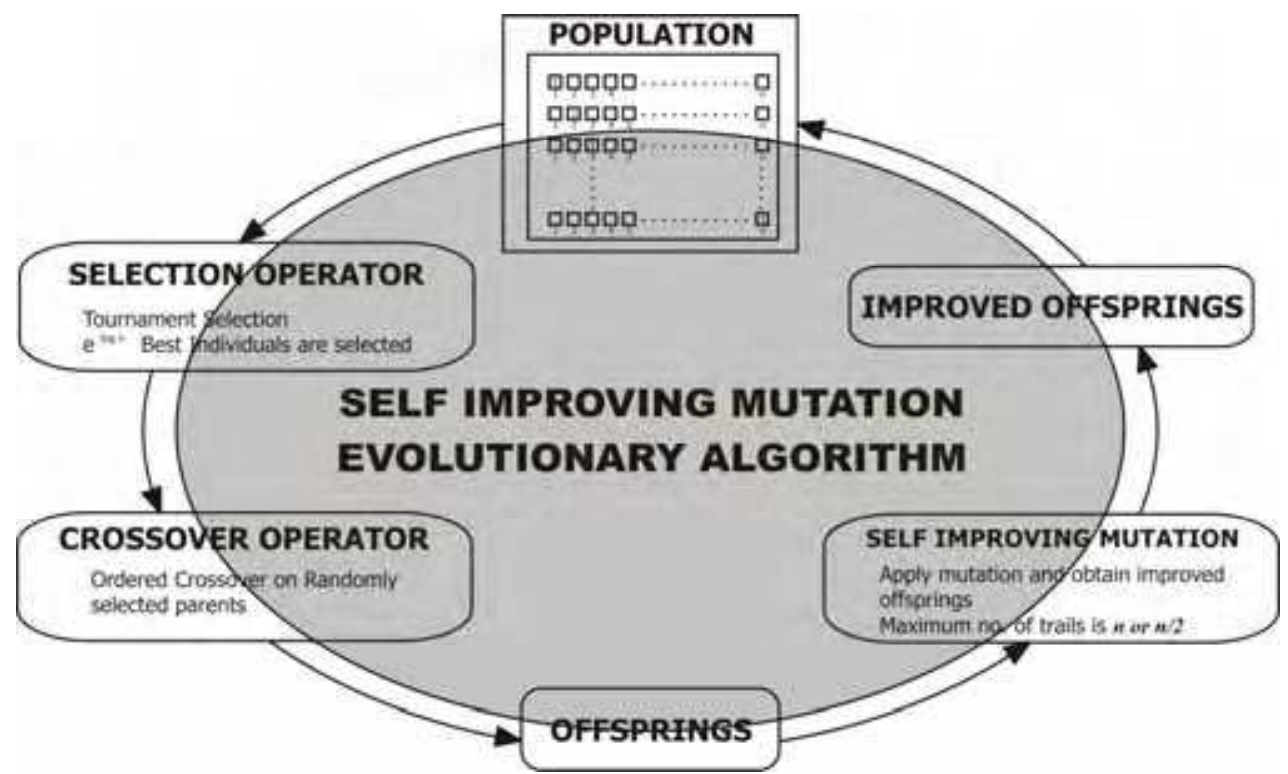

Figure 8. Self Improving Mutation Evolutionary Algorithm 


\subsubsection{Sequence Representation for SIMEA}

The solution representation for SIMEA is similar to the ILIEA. The sequence is mapped into a chromosome with the alleles assuming different and non repeating integer values in the $[1$, $n]$ interval. Any sequence can be mapped into this permutation representation. The objective function namely the total weighted tardiness of a sequence is considered as the fitness function of SIMEA.

\subsubsection{Initial Parents}

For the SIMEA, the size of the initial population is assumed to be the number of jobs. The individuals in the population are generated by means of a spread heuristics which ensures a better range of possible values of the chromosomes in the initial population. The individuals are generated in such a way that job 1 is fixed at the $n^{\text {th }}$ position for the $n^{\text {th }}$ chromosome.

\subsubsection{Selection Operator}

In this algorithm, it is proposed to use tournament selection with two different criteria on number of individuals selected for evolution (POP). In one version of SIMEA, all individuals in the population are selected for evolution (SIMEA I). Another version SIMEA applies a log arithmetic reduction heuristic, which allows only $e^{\log _{10} n}$ individuals are selected for evolution (SIMEA II).

\subsubsection{Crossover Operator}

On the selected individuals, the ordered crossover $(O X)$ is implemented. The $O X$ explained in the section 4.2.2 is used to generate offspring. Since, the number of individuals selected for evolution is more than two; more number of offspring will be generated.

\subsubsection{Self Improving Mutation}

The off springs obtained from the crossover are improved further by means of the self improving operator explained in section 4.2.3. Here, it is assumed to have the termination criterion for the improvement as $n / 2$.

\subsubsection{Termination Criterion}

The termination criterion of the algorithm is based on the number of predetermined number of generations. To have determined complexity, it is assumed to have $n^{2}$ number of generations as termination criteria for both SIMEA I E SIMEA II.

\section{Performance Evaluation}

The set of bottleneck facility total weighted tardiness problem instances available in the Operation Research Library maintained by Beasley are considered. The problem instances are generated as follows:

For each job $i(i=1, \ldots, n)$, an integer processing time $p_{i}$ was generated from the uniform distribution [1,100] and integer processing weight $w_{i}$ was generated from the uniform distribution $[1,10]$. Instance classes of varying hardness were generated by using different uniform distributions for generating the due dates. For a given relative range of due dates $R D D(R D D=0.2,0.4,0.6,0.8,1.0)$ and a given average tardiness factor $T F(T F=0.2,0.4,0.6$, 
$0.8,1.0)$, an integer due date $d_{i}$ for each job $i$ was randomly generated from the uniform distribution $[P x(1-T F-R D D / 2), P x(1-T F+R D D / 2)]$, where $P=\sum_{i=1}^{n} p_{i}$.

Here, there are 25 different combinations for $(R D D, T F)$ pairs and five replicates are taken for each $(R D D, T F)$ combinations yielding 125 different test instances for each value of $n$. In the OR library, there are three files $w t 40, w t 50$, and $w t 100$ containing the instances of size 40, 50, and 100 respectively. Each file contains the data for 125 instances, listed one after the other. The $n$ processing times are listed first, followed by the $n$ weights, and finally $n$ due dates, for each of the 125 instances in turn.

For example in $w t 40$ the first 40 integers in the file are the processing times for the 40 jobs in the first instance. The next 40 integers are the first instance's weights. The next 40 integers are the first instance's due dates. The next 40 integers are the second instance's processing times, etc.

\subsection{Optimal and Best Known Solution Values for SMTWTP}

Optimal values of solutions are available for 124 instances out of 125 problems for 40 jobs problem and the unsolved 40 jobs problem is number 19. The values for the unsolved problems given in the files wtopt40 is the best known to Crauwels, et. al., 1998.

Optimal values of solutions are available for 115 instances out of 125 problems the 50 jobs problem instances and the unsolved 50 jobs problems are problem no. 11, 12, 14, 19, 36, $44,66,87,88$ and 111 . The values for the unsolved problems given in the files wtopt50 are the best known to Crauwels, Potts \& Van Wassenhove. The values of the solutions not known to optimality have not been improved upon since and appear quite likely to be optimal.

The best solution values known to Crauwels, Potts \& Van Wassenhove (1998) for the 100 jobs problems are given in file wtbest100a. These solution values were used as the best known by both Crauwels et al. and Congram et al, 1990. Therefore using the best solution values known to Crauwels et al. allows results from future heuristics to be compared directly with the tables given.

The local search heuristic iterated dynasearch has in some cases found better solutions to the 100 job problems than those known by Crauwels, Potts \& Van Wassenhove. The best known solutions to date are given in the file wtbest $100 b$.

All the 125 problem instances for the different sizes $n=40, n=50$ and $n=100$ are solved by the three hybrid algorithms and compared with the best known results.

\subsection{Performance Analysis of Heuristic Improvement Algorithm}

Greedy forward heuristic is applied on $B H$ sequence to improve the solution. This is only a heuristic improvement operating on the sequence generated by the $B H$ as initial sequence. The average total weighted tardiness values calculated by the heuristic improvement for $n=$ 40 is 38809.91 , for $n=50$ is 54509.62 . But this heuristic improvement algorithm is not giving good results for higher size $n=100$. The results obtained are compared with the optimum/best known results available in OR library. The average total weighted tardiness for the different combinations of $(R D D, T F)$ are calculated and the percentage of deviation from best known values are given in table 1 for $n=40$ and for $n=50$. 


\begin{tabular}{|c|c|c|c|c|c|c|c|c|}
\hline \multirow{3}{*}{ S.No } & \multirow{3}{*}{ RDD } & \multirow{3}{*}{ T.F. } & \multicolumn{3}{|c|}{$\mathrm{n}=40$} & \multicolumn{3}{|c|}{$\mathrm{n}=50$} \\
\hline & & & \multicolumn{2}{|c|}{$\begin{array}{l}\text { Average weighted } \\
\text { tardiness }\end{array}$} & \multirow{2}{*}{$\begin{array}{c}\% \text { of } \\
\text { deviation }\end{array}$} & \multicolumn{2}{|c|}{$\begin{array}{l}\text { Average weighted } \\
\text { tardiness }\end{array}$} & \multirow{2}{*}{$\begin{array}{c}\% \text { of } \\
\text { deviation }\end{array}$} \\
\hline & & & Best Known & HIA & & Best Known & HIA & \\
\hline 1. & 0.2 & 0.2 & 1151.8 & 1252 & 8.699 & 2184.4 & 2335.6 & 6.922 \\
\hline 2. & 0.2 & 0.4 & 9221.2 & 9897.8 & 7.337 & 13343.4 & 14007 & 4.973 \\
\hline 3. & 0.2 & 0.6 & 21464.8 & 22612.4 & 5.346 & 43196.8 & 44285.6 & 2.521 \\
\hline 4. & 0.2 & 0.8 & 73120.2 & 76097.8 & 4.072 & 87714.4 & 91441.6 & 4.249 \\
\hline 5. & 0.2 & 1.0 & 112514 & 114099 & 1.409 & 189113 & 190486.6 & 0.726 \\
\hline 6. & 0.4 & 0.2 & 66.4 & 89.4 & 34.639 & 176.4 & 265 & 50.227 \\
\hline 7. & 0.4 & 0.4 & 4815.8 & 5459 & 13.356 & 6452.4 & 6999 & 8.471 \\
\hline 8. & 0.4 & 0.6 & 20039.8 & 21438.2 & 6.978 & 32574.6 & 35494.2 & 8.963 \\
\hline 9. & 0.4 & 0.8 & 69790.8 & 74849 & 7.248 & 89835.2 & 93276.8 & 3.831 \\
\hline 10. & 0.4 & 1.0 & 91736.8 & 92656.2 & 1.002 & 166049.6 & 168238.2 & 1.318 \\
\hline 11. & 0.6 & 0.2 & 0 & 34.8 & ----- & 0 & 39.2 & ----- \\
\hline 12. & 0.6 & 0.4 & 3273.6 & 3611.2 & 10.313 & 3426.6 & 4324.8 & 26.213 \\
\hline 13. & 0.6 & 0.6 & 18541.2 & 19754.8 & 6.545 & 23277.6 & 26031.8 & 11.832 \\
\hline 14. & 0.6 & 0.8 & 71892.4 & 73419.8 & 2.124 & 81545.4 & 84014.2 & 3.028 \\
\hline 15. & 0.6 & 1.0 & 90276 & 91539.6 & 1.400 & 130365 & 133429.2 & 2.351 \\
\hline 16. & 0.8 & 0.2 & 0 & 0 & 0.000 & 0 & 0 & 0.000 \\
\hline 17. & 0.8 & 0.4 & 609.4 & 1071.4 & 75.812 & 2191.2 & 2782 & 26.962 \\
\hline 18. & 0.8 & 0.6 & 14593.8 & 16380.8 & 12.245 & 25873.8 & 29013.6 & 12.135 \\
\hline 19. & 0.8 & 0.8 & 49719.8 & 51182.6 & 2.942 & 63134.6 & 65413.8 & 3.610 \\
\hline 20. & 0.8 & 1.0 & 121667.6 & 123609 & 1.600 & 153155.6 & 155049.4 & 1.234 \\
\hline 21. & 1.0 & 0.2 & 0 & 0 & 0.000 & 0 & 0 & 0.000 \\
\hline 22. & 1.0 & 0.4 & 774 & 960.2 & 24.057 & 1839.4 & 2074.6 & 12.787 \\
\hline 23. & 1.0 & 0.6 & 22629.2 & 24172.8 & 6.821 & 20864.8 & 23921.4 & 14.650 \\
\hline 24. & 1.0 & 0.8 & 51664 & 53565.4 & 3.680 & 76158 & 77863.4 & 2.239 \\
\hline 25. & 1.0 & 1.0 & 91482.4 & 92494.6 & 1.106 & 109855.4 & 111953.4 & 1.910 \\
\hline
\end{tabular}

Table 1. (RDD, TF) factor wise comparison - HIA

Experience with this method showed that in most instances the best sequence is obtained either immediately after the application of the backward phase or with a very few additional iterations of the forward phase. This seemed to be promising but not for large number of jobs.

\subsection{Performance Analysis of ILIEA}

The iterated local improvement algorithm is coded in $\mathrm{C}++$ on a personal computer with 1.3 $\mathrm{GHz}$ Pentium IV CPU and $128 \mathrm{MB}$ main memory and running on Micro soft Windows operating system 2000 (5 RELEASE version) with Borland C/C++ compiler (version 3.1). They are tested on 125 bench mark instances of total weighted tardiness problems of each sizes $n=40, n=50$ and $n=100$.

Here, there are 25 different combinations for $(R D D, T F)$ pairs and five replicates are taken for each $(R D D, T F)$ combinations yielding 125 different test instances for each value of $n$. 
The average total weighted tardiness values of five replicates of each (RDD, TF) combinations for the size $n=40, n=50, n=100$ are considered and compared with the best known values available in the file wtopt40, wtopt50, wtopt100 respectively.

The $(R D D, T F)$ factor wise comparison of results of iterated local improvement evolutionary algorithm as given in the table 2 .

\begin{tabular}{|c|c|c|c|c|c|c|c|c|c|c|c|}
\hline \multirow{3}{*}{ S.No } & \multirow{3}{*}{ RDD } & \multirow{3}{*}{ T.F. } & \multicolumn{3}{|c|}{$\mathrm{n}=40$} & \multicolumn{3}{|c|}{$\mathrm{n}=50$} & \multicolumn{3}{|c|}{$\mathrm{n}=100$} \\
\hline & & & \multicolumn{2}{|c|}{$\begin{array}{l}\text { Average } \\
\text { weighted } \\
\text { tardiness }\end{array}$} & \multirow{2}{*}{$\begin{array}{c}\% \text { of } \\
\text { deviation }\end{array}$} & \multicolumn{2}{|c|}{$\begin{array}{l}\text { Average } \\
\text { weighted } \\
\text { tardiness }\end{array}$} & \multirow{2}{*}{$\begin{array}{c}\% \text { of } \\
\text { deviation }\end{array}$} & \multicolumn{2}{|c|}{$\begin{array}{l}\text { Average } \\
\text { weighted } \\
\text { tardiness }\end{array}$} & \multirow{2}{*}{$\begin{array}{c}\% \text { of } \\
\text { deviation }\end{array}$} \\
\hline & & & \begin{tabular}{|c|} 
Best \\
known \\
value
\end{tabular} & ILIEA & & \begin{tabular}{|c|} 
Best \\
known \\
value
\end{tabular} & ILIEA & & \begin{tabular}{|c|} 
Best \\
known \\
value
\end{tabular} & ILIEA & \\
\hline 1. & 0.2 & 0.2 & 1151.8 & 1190.6 & 3.370 & 2184.4 & 2214.2 & 1.362 & 5343.8 & 6180.4 & 15.656 \\
\hline 2. & 0.2 & 0.4 & 9221.2 & 9221.2 & 0.000 & 13343.4 & 13523.2 & 1.347 & 52570 & 53164.6 & 1.131 \\
\hline 3. & 0.2 & 0.6 & 21464.8 & \begin{tabular}{|l|}
21464.8 \\
\end{tabular} & 0.000 & 43196.8 & 43216.8 & 0.004 & 185027.8 & \begin{tabular}{|l|}
185835.2 \\
\end{tabular} & 0.004 \\
\hline 4. & 0.2 & 0.8 & 73120.2 & 73120.2 & 0.000 & 87714.4 & 87749.4 & 0.004 & 433824.6 & 436382.6 & 0.006 \\
\hline 5. & 0.2 & 1.0 & 112514 & \begin{tabular}{|l|}
112514 \\
\end{tabular} & 0.000 & 1891131 & 189950.8 & 0.004 & 665021.4 & \begin{tabular}{|l|}
666331.8 \\
\end{tabular} & 0.002 \\
\hline 6. & 0.4 & 0.2 & 66.4 & 66.4 & 0.000 & 176.4 & 176.4 & 0.000 & 256.6 & 256.6 & 0.000 \\
\hline 7. & 0.4 & 0.4 & 4815.8 & 4833.2 & 0.360 & 6452.4 & 7102.2 & 10.070 & 24792.8 & 27262.8 & 9.963 \\
\hline 8. & 0.4 & 0.6 & 20039.8 & 20070 & 0.001 & 32574.6 & 32588.6 & 0.000 & 132402.4 & 137293.2 & 3.694 \\
\hline 9. & 0.4 & 0.8 & \begin{tabular}{|l|}
69790.8 \\
\end{tabular} & 69999 & 0.003 & \begin{tabular}{|l|}
89835.2 \\
\end{tabular} & \begin{tabular}{|l|}
90302.8 \\
\end{tabular} & 0.005 & 374993.8 & 379095.6 & 1.093 \\
\hline 10. & 0.4 & 1.0 & 91736.8 & \begin{tabular}{|l|}
91887.2 \\
\end{tabular} & 0.002 & 166049.6 & 166274 & 0.001 & 691626.8 & 703858.2 & 1.768 \\
\hline 11. & 0.6 & 0.2 & 0 & 0 & 0.000 & \begin{tabular}{|l|}
0 \\
\end{tabular} & 0 & 0.000 & 0 & 0 & 0.000 \\
\hline 12. & 0.6 & 0.4 & 3273.6 & 3303.4 & 0.009 & 3426.6 & 3604.6 & 0.052 & 12955 & \begin{tabular}{|l|}
14756 \\
\end{tabular} & 13.903 \\
\hline 13. & 0.6 & 0.6 & 18541.2 & 18583 & 0.002 & 23277.6 & 24065.2 & 0.034 & 85544.2 & \begin{tabular}{|c|}
91407.6 \\
\end{tabular} & 6.854 \\
\hline 14. & 0.6 & 0.8 & 71892.4 & 72006.8 & 0.002 & 81545.4 & 81756.4 & 0.003 & 315179.2 & 330526.8 & 4.869 \\
\hline 15. & 0.6 & 1.0 & 90276 & \begin{tabular}{|l|}
90796.6 \\
\end{tabular} & 0.006 & 130365 & 130731 & 0.003 & 607101.8 & 611426.4 & 0.007 \\
\hline 16. & 0.8 & 0.2 & 0 & 0 & 0.000 & 0 & 0 & 0.000 & 0 & \begin{tabular}{|l|}
0 \\
\end{tabular} & 0.000 \\
\hline 17. & 0.8 & 0.4 & 609.4 & 633.8 & 4.00 & 2191.2 & 2291.8 & 4.591 & 656.6 & 695.4 & 5.909 \\
\hline 18. & 0.8 & 0.6 & 14593.8 & 14672 & 0.005 & \begin{tabular}{|l|}
25873.8 \\
\end{tabular} & 26188.8 & 1.217 & 67259.2 & \begin{tabular}{|l|}
71899.8 \\
\end{tabular} & 6.900 \\
\hline 19. & 0.8 & 0.8 & 49719.8 & \begin{tabular}{|l|}
50817.2 \\
\end{tabular} & 2.207 & 63134.6 & 63179.8 & 0.001 & 295368.4 & 297195.6 & 0.006 \\
\hline 20. & 0.8 & 1.0 & 121667.6 & 121667.6 & 0.000 & 153155.61 & 153227.6 & 0.000 & 576902 & 578342.4 & 0.002 \\
\hline 21. & 1.0 & 0.2 & 0 & \begin{tabular}{|l|}
0 \\
\end{tabular} & 0.000 & \begin{tabular}{|l|}
0 \\
\end{tabular} & \begin{tabular}{l|}
0 \\
\end{tabular} & 0.000 & 0 & \begin{tabular}{|l|}
0 \\
\end{tabular} & 0.000 \\
\hline 22. & 1.0 & 0.4 & 774 & 780.4 & 0.008 & 1839.4 & 1839.4 & 0.000 & 285 & 338.4 & 18.736 \\
\hline 23. & 1.0 & 0.6 & 22629.2 & 22839.6 & 0.009 & 20864.8 & 21067.6 & 0.010 & 132623 & 141838.2 & 6.948 \\
\hline 24. & 1.0 & 0.8 & 51664 & \begin{tabular}{|l|}
51664 \\
\end{tabular} & 0.000 & \begin{tabular}{|l|}
76158 \\
\end{tabular} & \begin{tabular}{|l|}
76166.2 \\
\end{tabular} & 0.000 & 300435 & 303187.6 & 0.009 \\
\hline 25. & 1.0 & 1.0 & 91482.4 & \begin{tabular}{|l|}
91502.8 \\
\end{tabular} & 0.000 & 109855.41 & 109908.6 & 0.000 & 486114.2 & \begin{tabular}{|l|}
487220.8 \\
\end{tabular} & 0.002 \\
\hline
\end{tabular}

Table 2. (RDD, TF) factor wise comparison - ILIEA

From the table 2, it is observed that the average percentage of deviation is $0.399 \%$ from the best known values for size $n=40 ; 0.748 \%$ for size $n=50 ; 3.898 \%$ for size $n=100$.

\subsection{Performance Analysis of SIMEA I}

The SIMEA I algorithm has been implemented in the $\mathrm{C}++$ language on a personal computer with $1.3 \mathrm{GHz}$ Pentium IV CPU and $128 \mathrm{MB}$ main memory. The 
Sself Improving Evolutionary algorithm was running on FreeBSD operating system $(4.3$ RELEASE version) with the GNU C/C++ compiler (version 2.95.3) which is easier for CPU calculations. SIMEA $I$ is having the following parameters $P O P=n, M=n / 2$ and no. of iterations for termination is $n^{*} n$. The algorithm is tested on 125 bench mark instances of total weighted tardiness problems of each sizes $n=40, n=50$ and $n=100$.

The $(R D D, T F)$ factor wise comparison of results of Self Improving Evolutionary algorithm algorithm version $\mathrm{I}$ is given in the table 3.

\begin{tabular}{|c|c|c|c|c|c|c|c|c|c|c|c|}
\hline \multirow{3}{*}{ S.No } & \multirow{3}{*}{ RDD } & \multirow{3}{*}{ T.F. } & \multicolumn{3}{|c|}{$\mathrm{n}=40$} & \multicolumn{3}{|c|}{$n=50$} & \multicolumn{3}{|c|}{$\mathrm{n}=100$} \\
\hline & & & \multicolumn{2}{|c|}{$\begin{array}{l}\text { Average } \\
\text { weighted } \\
\text { tardiness }\end{array}$} & \multirow{2}{*}{$\begin{array}{c}\% \text { of } \\
\text { deviation }\end{array}$} & \multicolumn{2}{|c|}{$\begin{array}{l}\text { Average } \\
\text { weighted } \\
\text { tardiness }\end{array}$} & \multirow{2}{*}{$\begin{array}{c}\% \text { of } \\
\text { deviation }\end{array}$} & \multicolumn{2}{|c|}{$\begin{array}{l}\text { Average } \\
\text { weighted } \\
\text { tardiness } \\
\end{array}$} & \multirow{2}{*}{$\begin{array}{c}\% \text { of } \\
\text { deviation }\end{array}$} \\
\hline & & & $\begin{array}{c}\text { Best } \\
\text { known } \\
\text { value } \\
\end{array}$ & SIMEA I & & \begin{tabular}{|c|} 
Best \\
known \\
value
\end{tabular} & SIMEA I & & $\begin{array}{c}\text { Best } \\
\text { known } \\
\text { value }\end{array}$ & SIMEA I & \\
\hline 1. & 0.2 & 0.2 & 1151.8 & \begin{tabular}{|l|l|}
1170.4 \\
\end{tabular} & 1.615 & 2184.4 & 2224.8 & 1.849 & 5343.8 & 5372 & 0.528 \\
\hline 2. & 0.2 & 0.4 & 9221.2 & 9315 & 1.017 & 13343.4 & 13538.2 & 1.460 & 52570 & 52801.2 & 0.440 \\
\hline 3. & 0.2 & 0.6 & 21464.8 & 21575.6 & 0.516 & 43196.8 & 43458.8 & 0.607 & 185027.8 & 185742.8 & 0.386 \\
\hline 4. & 0.2 & 0.8 & 73120.2 & 73223.8 & 0.142 & 87714.4 & 87981.2 & 0.304 & 433824.6 & 434668.6 & 0.195 \\
\hline 5. & 0.2 & 1.0 & 112514 & 112539.6 & 0.023 & 189113 & 189139 & 0.014 & 665021.4 & \begin{tabular}{|l|}
665064 \\
\end{tabular} & 0.006 \\
\hline 6. & 0.4 & 0.2 & 66.4 & 66.4 & 0.000 & 176.4 & 195.8 & 10.998 & 256.6 & \begin{tabular}{|l|}
280.4 \\
\end{tabular} & 9.275 \\
\hline 7. & 0.4 & 0.4 & 4815.8 & 4892.8 & 1.599 & 6452.4 & 6599.4 & 2.278 & 24792.8 & 25229.2 & 1.760 \\
\hline 8. & 0.4 & 0.6 & 20039.8 & 20180 & 0.670 & 32574.6 & 32968.2 & 1.208 & 132402.4 & 133846 & 1.090 \\
\hline 9. & 0.4 & 0.8 & \begin{tabular}{|l|}
69790.8 \\
\end{tabular} & 70047.2 & 0.367 & \begin{tabular}{|l|}
89835.2 \\
\end{tabular} & 90117 & 0.314 & 374993.8 & 376054.2 & 0.283 \\
\hline 10. & 0.4 & 1.0 & 91736.8 & 91806 & 0.075 & 166049.6 & 166105.4 & 0.034 & 691626.8 & \begin{tabular}{|l|}
691788 \\
\end{tabular} & 0.023 \\
\hline 11. & 0.6 & 0.2 & $\begin{array}{l}0 \\
\end{array}$ & 0 & 0.000 & \begin{tabular}{|l|}
0 \\
\end{tabular} & \begin{tabular}{l|}
0 \\
\end{tabular} & 0.000 & 0 & \begin{tabular}{|l|}
0 \\
\end{tabular} & 0.000 \\
\hline 12. & 0.6 & 0.4 & 3273.6 & 3420 & 4.472 & 3426.6 & 3518.8 & 2.691 & 12955 & \begin{tabular}{|l|}
13543.2 \\
\end{tabular} & 4.540 \\
\hline 13. & 0.6 & 0.6 & \begin{tabular}{|l|}
18541.2 \\
\end{tabular} & 19224.6 & 3.686 & 23277.6 & 23824.4 & 2.349 & 85544.2 & \begin{tabular}{|l|}
86340.4 \\
\end{tabular} & 0.931 \\
\hline 14. & 0.6 & 0.8 & 71892.4 & 71968.2 & 0.105 & 81545.4 & 81861 & 0.387 & 315179.2 & 316436.6 & 0.399 \\
\hline 15. & 0.6 & 1.0 & 90276 & 90349 & 0.081 & 130365 & 130433.6 & 0.053 & 607101.8 & 607239.6 & 0.023 \\
\hline 16. & 0.8 & 0.2 & 0 & 0 & 0.000 & 0 & \begin{tabular}{l|}
0 \\
\end{tabular} & 0.000 & \begin{tabular}{l|}
0 \\
\end{tabular} & \begin{tabular}{|l|}
0 \\
\end{tabular} & 0.000 \\
\hline 17. & 0.8 & 0.4 & 609.4 & 717.6 & 17.755 & 2191.2 & 2255.2 & 2.921 & 656.6 & 685.8 & 4.447 \\
\hline 18. & 0.8 & 0.6 & 14593.8 & 14845 & 1.721 & 25873.8 & 26231 & 1.381 & 67259.2 & \begin{tabular}{|l|}
68757 \\
\end{tabular} & 2.227 \\
\hline 19. & 0.8 & \begin{tabular}{|l|l|}
0.8 \\
\end{tabular} & 49719.8 & 49861 & 0.284 & 63134.6 & 63435.6 & 0.477 & 295368.4 & 296705.4 & 0.453 \\
\hline 20. & 0.8 & 1.0 & 121667.6 & 121714.4 & 0.038 & 153155.6 & 153222 & 0.043 & 576902 & 577189 & 0.050 \\
\hline 21. & 1.0 & 0.2 & 0 & 0 & 0.000 & \begin{tabular}{|l|}
0 \\
\end{tabular} & 0 & 0.000 & 0 & 0 & 0.000 \\
\hline 22. & 1.0 & 0.4 & 774 & 784 & 1.292 & 1839.4 & 1935.2 & 5.208 & 285 & 295 & 3.509 \\
\hline 23. & 1.0 & 0.6 & 22629.2 & 22975 & 1.528 & 20864.8 & 21290.2 & 2.039 & 132623 & 134451 & 1.369 \\
\hline 24. & 1.0 & 0.8 & 51664 & 51926.8 & 0.508 & \begin{tabular}{|l|}
76158 \\
\end{tabular} & 76405.6 & 0.325 & 300435 & 301911.8 & 0.489 \\
\hline 25. & 1.0 & 1.0 & 91482.4 & 100839.4 & 10.228 & 109855.4 & 110345.6 & 0.446 & 486114.2 & 486581.6 & 0.096 \\
\hline
\end{tabular}

Table 3. $(R D D, T F)$ factor wise comparison - SIMEA I

From the table 3 , it is observed that the average percentage of deviation is $1.91 \%$ from the best known values for size $n=40 ; 1.49 \%$ for size $n=50 ; 1.3 \%$ for size $n=100$. 


\subsection{Performance Comparision of SIMEA II}

The SIMEA II algorithm has also been implemented in the $\mathrm{C}++$ language on a personal computer with $1.3 \mathrm{GHz}$ Pentium IV CPU and $128 \mathrm{MB}$ main memory. SIMEA II is having the following parameters $P O P=e^{\log _{10} n}, M=n / 2$ and no. of iterations for termination is $n^{*} n$. The algorithm is tested on 125 bench mark instances of total weighted tardiness problems of each sizes $n=40, n=50$ and $n=100$.

The $(R D D, T F)$ factor wise comparison for the average total weighted tardiness SIMEA II with reduction heuristics and the percentage of deviation from the best known values are given in the table 4 .

\begin{tabular}{|c|c|c|c|c|c|c|c|c|c|c|c|}
\hline \multirow{3}{*}{ S.No } & \multirow{3}{*}{ RDD } & \multirow{3}{*}{ T.F. } & \multicolumn{3}{|c|}{$\mathrm{n}=40$} & \multicolumn{3}{|c|}{$\mathrm{n}=50$} & \multicolumn{3}{|c|}{$\mathrm{n}=100$} \\
\hline & & & \multicolumn{2}{|c|}{$\begin{array}{l}\text { Average } \\
\text { weighted } \\
\text { tardiness } \\
\end{array}$} & \multirow{2}{*}{$\begin{array}{c}\% \text { of } \\
\text { deviation }\end{array}$} & \multicolumn{2}{|c|}{$\begin{array}{c}\text { Average } \\
\text { weighted } \\
\text { tardiness }\end{array}$} & \multirow{2}{*}{$\begin{array}{c}\% \text { of } \\
\text { deviation }\end{array}$} & \multicolumn{2}{|c|}{\begin{tabular}{c|} 
Average \\
weighted \\
tardiness \\
\end{tabular}} & \multirow{2}{*}{$\begin{array}{c}\% \text { of } \\
\text { deviation }\end{array}$} \\
\hline & & & $\begin{array}{c}\text { Best } \\
\text { known } \\
\text { value }\end{array}$ & $\begin{array}{c}\text { SIMEA } \\
\text { II }\end{array}$ & & \begin{tabular}{|c|} 
Best \\
known \\
value
\end{tabular} & $\begin{array}{c}\text { SIMEA } \\
\text { II }\end{array}$ & & \begin{tabular}{|c|} 
Best \\
known \\
value
\end{tabular} & $\begin{array}{c}\text { SIMEA } \\
\text { II }\end{array}$ & \\
\hline 1. & 0.2 & 0.2 & 1151.8 & 1170 & 1.580 & 2184.4 & 2211.8 & & 5343.8 & 5371.4 & \\
\hline 2. & 0.2 & 0.4 & 9221.2 & 9369.4 & & \begin{tabular}{|l|}
13343.4 \\
\end{tabular} & 13363.8 & & 52570 & 52797 & \\
\hline 3. & 0.2 & 0.6 & 21464.8 & 21598 & 0.6 & \begin{tabular}{|l|}
43196.8 \\
\end{tabular} & 43540.6 & 796 & \multicolumn{2}{|c|}{185027.8185655 .2} & 0.339 \\
\hline 4. & 0.2 & 0.8 & 73120.2 & 73824.4 & & 87714.4 & 88120.8 & 0.463 & \multicolumn{2}{|c|}{\begin{tabular}{|l|l|}
433824.6 & 434416 \\
\end{tabular}} & 136 \\
\hline 5. & 0.2 & 1.0 & 112514 & 112769 & 0.227 & 189113 & 189373.2 & 138 & \multicolumn{2}{|c|}{\begin{tabular}{|l|l|}
665021.4 & 665842 \\
\end{tabular}} & 123 \\
\hline 6. & 0.4 & 0.2 & \begin{tabular}{|l|}
66.4 \\
\end{tabular} & 120.8 & 81. & 176.4 & 212 & 20.181 & 256.6 & 313.2 & 22.058 \\
\hline 7. & 0.4 & 0.4 & 4815.8 & 4905.4 & & 6452.4 & 6712.6 & & 24792.8 & 25412.8 & \\
\hline 8. & 0.4 & 0.6 & 20039.8 & 20345.6 & & 32574.6 & 32913 & 39 & 132402.4 & 134384.2 & 1.497 \\
\hline 9. & 0.4 & 0.8 & 69790.8 & 70228 & & 89835.2 & 91501 & 54 & & 809 \\
\hline 10. & 0.4 & 1.0 & 91736.8 & 92310.6 & 0.625 & \multicolumn{2}{|c|}{166049.6166540 .8} & 0.296 & \multicolumn{2}{|c|}{\begin{tabular}{|l|l|}
691626.8 & 693124 \\
\end{tabular}} & 0.216 \\
\hline 11. & 0.6 & 0.2 & \begin{tabular}{|l|}
0 \\
\end{tabular} & 0 & & \begin{tabular}{|l|}
0 \\
\end{tabular} & \begin{tabular}{|l|}
0 \\
\end{tabular} & 000 & 0 & 0 & 000 \\
\hline 12. & 0.6 & 0.4 & 3273.6 & 3575.4 & & 3426.6 & 3745 & 92 & 12955 & 13465.2 & \\
\hline 13. & 0.6 & 0.6 & 18541.2 & 18714.4 & & 23277.6 & 24133.6 & & 85544.2 & 87208.4 & \\
\hline 14. & 0.6 & 0.8 & 71892.4 & 72350.2 & 0.637 & \begin{tabular}{|l|}
81545.4 \\
\end{tabular} & 82350 & 987 & \multicolumn{2}{|c|}{315179.2316216 .4} & 0.329 \\
\hline 15. & 0.6 & 1.0 & 90276 & 90897 & 0.6 & 130365 & 130864 & 0.383 & \multicolumn{2}{|c|}{607101.8608054 .2} & 0.157 \\
\hline 16. & 0.8 & 0.2 & 0 & 0 & & $\begin{array}{l}0 \\
\end{array}$ & 0 & 0.000 & 0 & \begin{tabular}{|l|}
0 \\
\end{tabular} & 0.000 \\
\hline 17. & 0.8 & 0.4 & 609.4 & 837 & & 2191.2 & 2439 & 309 & 656.6 & 1065.2 & 62.230 \\
\hline 18. & 0.8 & 0.6 & 14593.8 & 15030.4 & 2.992 & \begin{tabular}{|l|}
25873.8 \\
\end{tabular} & 26446.8 & 2.215 & 67259.2 & 69316.8 & .059 \\
\hline 19. & 0.8 & 0.8 & 49719.8 & 50249.2 & 65 & 63134.6 & 63622.4 & 773 & 295368.4 & 296488 & 0.379 \\
\hline 20. & 0.8 & 1.0 & 121667.6 & 121976 & 0.253 & 153155.6 & 153291 & 0.088 & 576902 & 577365.6 & 0.080 \\
\hline 21. & 1.0 & 0.2 & 0 & 0 & & \begin{tabular}{l|} 
\\
\end{tabular} & 0 & 0.000 & 0 & \begin{tabular}{l|}
0 \\
\end{tabular} & 000 \\
\hline 22. & 1.0 & 0.4 & 774 & 1111.6 & 43.618 & \begin{tabular}{|l|}
1839.4 \\
\end{tabular} & 1973 & 7.263 & 285 & 310.4 & 8.912 \\
\hline 23. & 1.0 & 0.6 & 22629.2 & 23411.2 & 3.456 & \begin{tabular}{|l|}
20864.8 \\
\end{tabular} & 22067.6 & 5.765 & 132623 & 135687 & 2.301 \\
\hline 24. & 1.0 & 0.8 & 51664 & 52064.6 & & \begin{tabular}{|l|}
76158 \\
\end{tabular} & 77737.6 & 2.074 & 300435 & 301742.4 & 0.433 \\
\hline 25. & 1.0 & 1.0 & 91482.4 & 92003.4 & 0.570 & 109855.4 & 110337.6 & 0.439 & 486114.2 & 487448.4 & 0.274 \\
\hline
\end{tabular}

Table 4. (RDD, TF) factor wise comparison - SIMEA II

From the table 4 , it is observed that the average percentage of deviation is $7.724 \%$ from the best known values for size $n=40 ; 2.978 \%$ for size $n=50$ and $4.506 \%$ for size $n=100$. 


\subsection{Performance Comparison of Algorithms}

The average total weighted tardiness of all 125 problem instances obtained by different search algorithms are calculated for the different sizes $n=40, n=50 \mathcal{E} n=100$ and compared with the best known values and given in table 5 .

\begin{tabular}{|l|l|l|l|l|l|l|l|}
\hline S.No & $n$ & $\begin{array}{l}\text { Best } \\
\text { known } \\
\text { values }\end{array}$ & $\begin{array}{l}\text { Backward } \\
\text { Heuristics }\end{array}$ & HIA & ILIEA & $\begin{array}{l}\text { SIMEA } \\
\text { I }\end{array}$ & $\begin{array}{l}\text { SIMEA } \\
\text { II }\end{array}$ \\
\hline 1 & 40 & 37641.8 & 52602.07 & 38809.91 & 37745.35 & 38137.67 & 37954.46 \\
\hline 2 & 50 & 52893.1 & 74157.74 & 54509.62 & 53086.02 & 53083.44 & 53339.87 \\
\hline 3 & 100 & 217852.1 & 314076.6 & $\begin{array}{l}\text { Code Not } \\
\text { Structured }\end{array}$ & 220978.9 & 218439.3 & 218788.4 \\
\hline
\end{tabular}

Table 5. Comparison of Average Total weighted tardiness values

The percentage of deviation of the average total weighted tardiness obtained by the different algorithms are calculated and given in figure 9.

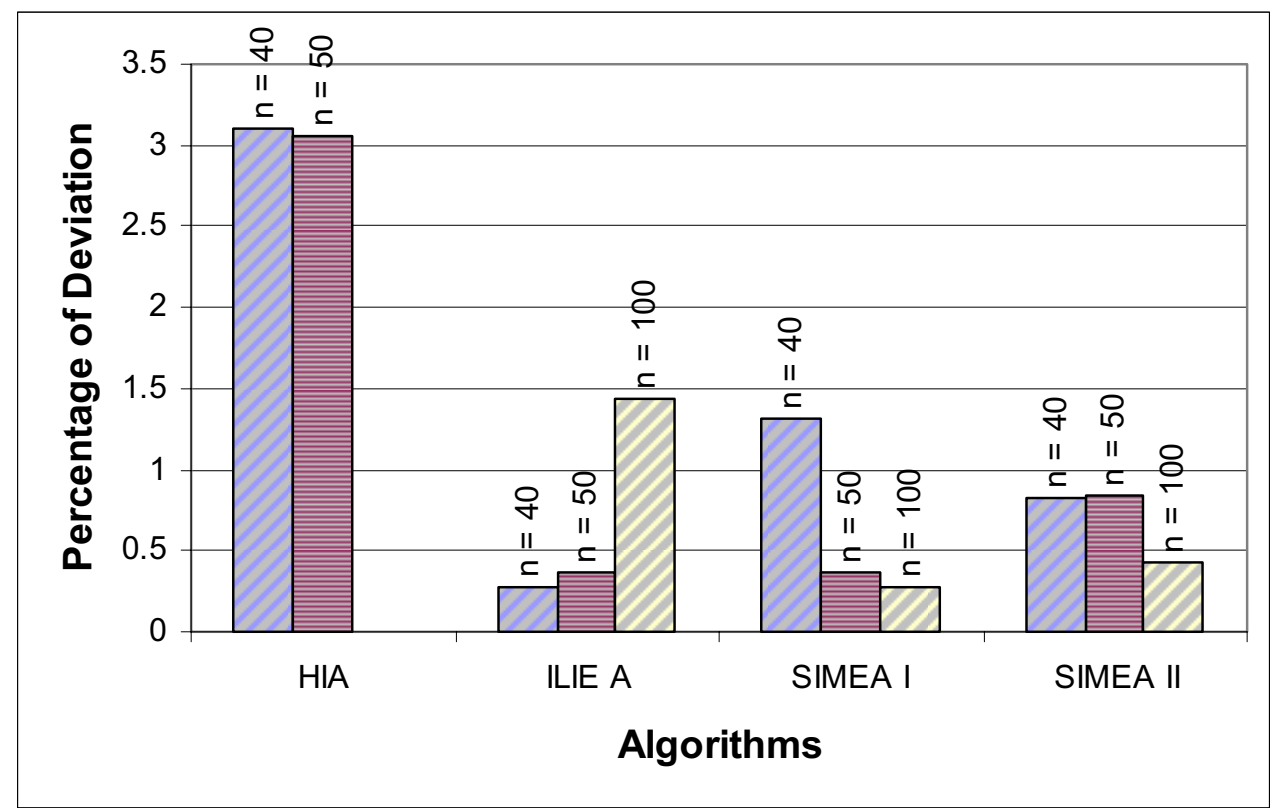

Figure 9. Performance Comparison

From the figures, it is experienced that the performance of heuristic improvement algorithm is poor for the higher sizes of $n$. This algorithm is giving results within less computational time and it is not able to solve the problems of size $n=100$ effectively and so not included in the figure 9.

The iterated local improvement algorithm is giving results closer to the best known values for $n=40$ than other algorithms. But, when size of the problem is increased the percentage of deviation is also increasing. 
But self improving mutation evolutionary algorithms perform well for higher sizes of problems. It is observed SIMEA II is producing similar results with lesser computational time than SIMEA I.

\subsection{Percent Improvement}

Since all the three algorithms have been developed from the backward heuristic sequence, the percent improvement of the different heuristic search algorithms are calculated by the formula

$$
\text { Percent improvement }=\frac{Z_{\text {(backward phase) }}-Z_{(\text {algorithm) }}}{Z_{\text {(backward phase) }}} \times 100 \%
$$

The average percent improvement of the various heuristic search algorithms from backward phase heuristic for different sizes $n=40, n=50 \mathcal{E} n=100$ are given in the table 6 and comparison of percent improvement is shown in figure 10.

\begin{tabular}{|l|l|l|l|l|l|}
\hline S.No & $n$ & HIA & ILIEA & SIMEA I & SIMEA II \\
\hline 1. & 40 & 26.22 & 28.24 & 27.50 & 27.85 \\
\hline 2. & 50 & 26.49 & 28.41 & 28.41 & 28.07 \\
\hline 3. & 100 & NA & 29.64 & 30.45 & 30.33 \\
\hline
\end{tabular}

Table 6. Average Percent Improvement from Backward Heuristics

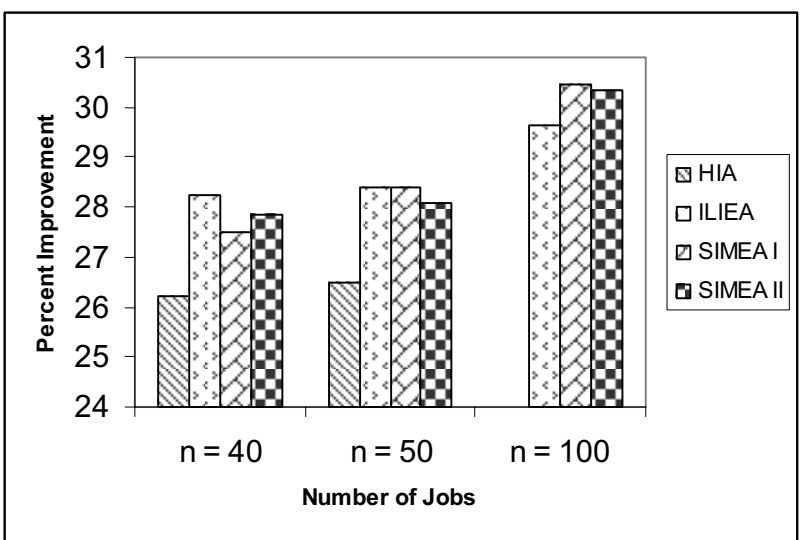

Figure 10. Comparison of Percent Improvement

The observations on percent improvement reveals that SIMEA I \& SIMEA II provide higher improvements than other two heuristic algorithm namely, HIA \& ILIEA. Besides this, HIA is not structured to solve problems of higher size (i.e. $n=100$ ).

\section{Conclusion}

Scheduling function is embedded in the domain of production planning control and it plays an important role in the manufacturing process. The bottleneck scheduling problems can arise in different practical situations in the manufacturing system. The objective function of scheduling problem may be minimization of make span, lateness, weighted measures etc. In 
weighted performance measure cases, the priority indexes may be given to different jobs according to the importance. Total weighted tardiness problems are proved to be NP hard type problems. Enumerative methods are time consuming to solve problems of higher sizes and construction heuristics are giving inaccurate results. In practice, there is a need to get near optimal solutions within reasonable time. Heuristic search algorithms are used to get near optimal solution. In this work, an attempt is made to hybridize trajectory and population methods for solving the bottleneck facility total weighted tardiness problems. The three heuristic search algorithms are developed and used to solve the different benchmark instances.

Heuristic improvement algorithm is a trajectory method operating on a single sequence developed by some construction heuristics as initial sequence. The forward heuristic is working by a heuristic procedure with interchange method. It is observed that the process of this improvement algorithm is tedious and is not able to solve problems of higher sizes

The ILIEA algorithm uses only a single pair of parents; one sequence obtained from a greedy backward phase heuristic and the other by random generation act as the initial parents. The performance of this algorithm, with and without crossover operation, is compared. The average percentage of deviation is ranging from $27.54 \%$ to $38.28 \%$ for the iterated local improvement algorithm without crossover and the ranging from $0.28 \%$ to $16.84 \%$ for the iterated local improvement evolutionary algorithm with crossover.

SIMEA I algorithm is the extended form of the iterated local improvement evolutionary algorithm with size of the population equal to number of jobs. Further, a log arithmetic reduction rule is applied in the parent selection to develop another version SIMEA II and tested with benchmark instances of SMTWTP. The performance of these two versions is compared and it is observed SIMEA II is producing similar results with lesser computational time.

\section{References}

Abdul-razaq T. S., Potts C. N. and VanWassenhove L. N. (1990), A survey of algorithms for the single-machine total weighted tardiness scheduling problem, Discrete Applied Mathematics, Vol. 26, pp. 235 - 253.

Ackley D.H. (1987), A connectionist machine for genetic hill climbing, Kluwer Academic Press, Boston.

Bahram Alidaee and Ramakrishnan K.R. (1996), A Computational Experiment of Covert-AU Class of Rules for Single Machine Tardiness Scheduling Problems, Computers Industrial Engineering Journal, Vol. 30, No. 2, pp 201 - 209.

Beasley J.E., O.R. library, http:/ / www.ms.ic.ac.uk/jeb/orlib/wtinfo.html.

Baker K.R. (1995), Elements of Sequencing and Scheduling, Amos Tuck School, Dartmouth College, Hanover, NH.

Blackstone J., Phillips D. and Hogg G. (1982), A state-of-the-art survey of dispatching rules for manufacturing job shop operations, International Journal of Production Research, Vol. 20, No. 1, pp. 27-45.

Congram R. K., Potts C. N. and Van de Velde S. L. (1998), An iterated dynasearch algorithm for the single machine total weighted tardiness scheduling problem, Technical report, Faculty of Mathematical Studies, University of Southampton, Southampton, UK.

Crauwels H. A. J., Potts C. N. and Van Wassenhove L. N. (1998), Local search heuristics for the single machine total weighted tardiness scheduling problem, INFORMS Journal on Computing, Vol. 10, No. 3, pp. 341 - 350. 
Della Croce F., Tadei R., Baracco P. and Grosso A. (1998), A New Decomposition Approach for the Single Machine Total Tardiness Scheduling Problem, Journal of the Operational Research Society, Vol. 49, pp. 1101- 1106.

Den Besten M. L., Stützle T., and Dorigo M. (2000), Ant colony optimization for the total weighted tardiness problem, In the proceedings Parallel Problem Solving from Nature: 6th international conference of Berlin, Marc Schoenauer, Kalyanmoy Deb, Günter Rudolph, Xin Yao, Evelyne Lutton, Juan Julian Merelo, and Hans-Paul Schwefel, editors, Springer Verlag. Vol.1917 of LNCS, pp. 611-620.

Deshpande A. S. and Triantaphyllou E. (1998), A Greedy Randomized Adaptive Search Procedure (GRASP) for Inferring Logical Clauses from Examples in Polynomial Time and some Extensions, Mathematical and Computer Modeling, Vol. 27, No. 1, pp. 75-99.

Dileep R. Sule (1997), Industrial Scheduling, PWS Publishing Company, An International Thomson Publishing Company.

Drobouchevitch G., Strusevich V.A. (2000), Heuristics for the two-stage job shop scheduling problem with a bottleneck machine, European Journal of Operational Research, Vol. 123, pp. 229-240.

Drobouchevitch I.G., Strusevich V.A. (2001), Two-stage open shop scheduling problem with a bottleneck machine, European Journal of Operational Research, Vol. 128, pp. 159-174.

Du J. and Leung J.Y.T. (1990), Minimizing Total Tardiness on One Machine is NP Hard, Mathematics of Operational Research, Vol. 15, pp. 483 - 495.

Feo T. A. and Resende M. G. C. (1995), Greedy Randomized Adaptive Search Procedures, Journal of Global Optimization, Vol. 6, pp. 109-133.

Glover F. (1989), Tabu Search. Part I, ORSA Journal on Computing, Vol. 1, pp. 190-206.

Glover F. (1990), Tabu Search. Part II, ORSA Journal on Computing, Vol. 2, pp. 4-32.

Glover F., Kelly J., and Laguna M. (1995), Genetic Algorithms and Tabu Search: Hybrids for Optimization, Computers and Operations Research, Vol. 22, No. 1, pp. 111-134.

Glover F., Taillard E., and de Werra D. (1993), A User's Guide to Tabu Search, Annals of Operations Research, Vol. 41, pp. 3-28.

Goldberg D. E. (1989), Genetic Algorithms in Search, Optimization and Machine Learning, Addison-Wesley, Reading, MA.

Haupt R. (1989), A Survey of Priority Rule-Based Scheduling, OR Spektrum Vol. 11, pp. 3- 16.

Helena R. Lourenco, Olivier Martin and Thomas Stutzle (2001), A Beginner's Introduction to Iterated Local Search, In the Proceedings of MIC'2001 - 4th Meta-heuristics International Conference at Porto, Portugal.

Helena Ramalhinho Lourenco (1995), Job Shop Scheduling: Computational Study of Local Search and Large-Step Optimization Methods, European Journal of Operational Research, Vol. 83, No. 2, pp. 347 - 364.

Kirkpatrick S., Gelatt Jr C. D. and Vecchi M. P. (1983), Optimization by Simulated Annealing, Science, Vol. 220, pp. 671-680.

Lawler E.L. (1977), A 'Pseudopolynomial' algorithm for sequencing jobs to minimize total tardiness, Annals of discrete Mathematics, Vol. 1, pp. 331-342.

Lawler E. L. and Wood D. E. (1966), Branch-and-bound methods: A survey, Operations Research, Vol. 14, No, 4, pp. 699-719.

Maheswaran. R and Ponnambalam S.G., (2003) An Investigation on Single Machine Total Weighted Tardiness Scheduling Problems, International Journal on Advanced Manufacturing Technology, Vol. 22, No. 3 - 4, pp. 243 - 248. 
Maheswaran. R., (2004), Heuristics Search Algorithms in Single Machine Scheduling, Unpublished Ph.D Thesis, Manonmanium Sundaranar University, Trinelveli.

Maheswaran. R and Ponnambalam S.G., (2005) An Intensive Search Evolutionary Algorithm for Single Machine Total Weighted Tardiness Scheduling Problems, International Journal on Advanced Manufacturing Technology, Vol. 25, No. 7 - 8, pp. 772 - 776.

Maheswaran. R, Ponnambalam S.G. and Aravindan C. (2005) A Meta-heuristic approach to Single Machine Scheduling Problems, International Journal on Advanced Manufacturing Technology, Vol. 26, No. 9 - 10, pp. 1150 - 1156.

Montazer M. and Van Wassenhove L. (1990), Analysis of scheduling rules for an FMS, International Journal of Production Research, Vol. 28, pp. 785-802.

Morton, T. E., Pentico, D. W. (1993), Heuristic Scheduling Systems; With Applications to Productions Systems and Project Management, John Wiley \& Sons Inc., New York, Chichester, Brisbane, Toronto, Singapore.

Osman I. H. (1996), Metaheuristics: A Bibliography, Annals of Operations Research, Vol. 63, pp. 513-623.

Osman I. H. and Kelly, J. P. (1996), Meta-Heuristics: An Overview, in Meta-Heuristics: Theory $\mathcal{E}$ Applications, Kluwer Academic Publishers, Boston/London/Dordrecht.

Panwalker S. and Iskander W. (1977), A survey of scheduling rules, Operations Research, Vol. 25, No. 1, pp. 45-61.

Paulo M. Franca, Alexandre Mendes and Pablo Moscato (2001), A Memetic Algorithm for the Total Tardiness Single Machine Scheduling Problem, European Journal of Operational Research, Vol. 132, No.1, pp 224 - 242.

Potts C.N. and Van Wassenhove L.N. (1982), A Decomposition Algorithm for the Single Machine Tardiness Problem, Operations Research Letters Vol. 32, pp. 177-181.

Potts C. N. and VanWassenhove L. N. (1985), A branch and bound algorithm for total weighted tardiness problem, Operations Research, Vol. 33, pp. 363 - 377.

Radcliffe N.J., and Surry P.D. (1994), Formal Memetic Algorithm, Evolutionary Computing, Selected Papers from AISB Workshop, Lecture Notes in Computer Science, Springer Verlag, pp. 1 - 16.

Reeves C. R. (1993), Modern Heuristic Techniques for Combinatorial Problems, Blackwell Scientific Publications, Oxford.

Schrage L.E. and Baker K.R. (1978), Dynamic Programming Solution of Sequencing Problems with Precedence Constraints, Operations Research Vol. 26, pp. 444-449.

Shapiro J. (1979), A survey of Lagrangian techniques for discrete optimization, Annals of Discrete Mathematics, Vol. 5, pp. 113-138.

Szwarc W. and Mukhopadhyay S. (1997), Decomposition of the Single Machine Total Tardiness Problem, Operations Research Letters, Vol. 19, pp. 243-250.

Starkweather. T., McDaniel. S., Whitley. C., Mathias. K., Whitley. D. (1991), A Comparison of Genetic Sequencing Operators, In the proceedings of the $4^{\text {th }}$ International Conference on Genetic Algorithms, San Diego, California, Morgan Kaufmann, publishers, pp. 69-76.

Thomas Baeck, Frank Hoffmeister and Hans Paul Schwefel, (1991), A survey of evolution strategies, In proceedings of the $4^{\text {th }}$ International Conference on Genetic Algorithms, Morgan Kaufmann, San Mateo.

Tsiushuang C., Xiangtong Q. and Fengsheng T. (1997), Single machine scheduling to minimize weighted earliness subject to maximum tardiness, Computers Operations Research, pp. 147-157. 


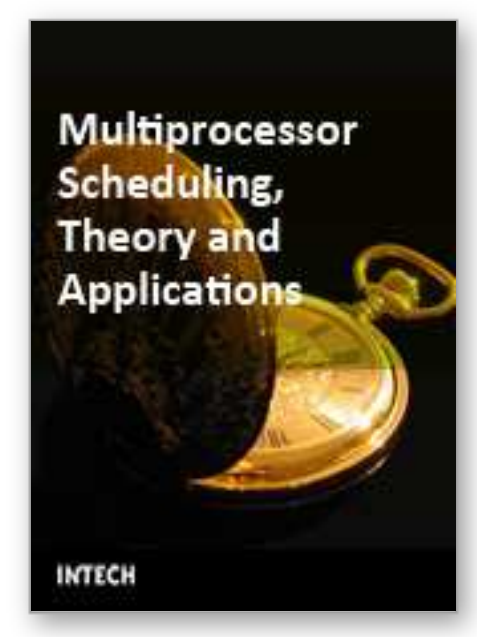

\author{
Multiprocessor Scheduling, Theory and Applications \\ Edited by Eugene Levner
}

ISBN 978-3-902613-02-8

Hard cover, 436 pages

Publisher I-Tech Education and Publishing

Published online 01, December, 2007

Published in print edition December, 2007

A major goal of the book is to continue a good tradition - to bring together reputable researchers from different countries in order to provide a comprehensive coverage of advanced and modern topics in scheduling not yet reflected by other books. The virtual consortium of the authors has been created by using electronic exchanges; it comprises 50 authors from 18 different countries who have submitted 23 contributions to this collective product. In this sense, the volume can be added to a bookshelf with similar collective publications in scheduling, started by Coffman (1976) and successfully continued by Chretienne et al. (1995), Gutin and Punnen (2002), and Leung (2004). This volume contains four major parts that cover the following directions: the state of the art in theory and algorithms for classical and non-standard scheduling problems; new exact optimization algorithms, approximation algorithms with performance guarantees, heuristics and metaheuristics; novel models and approaches to scheduling; and, last but least, several real-life applications and case studies.

\title{
How to reference
}

In order to correctly reference this scholarly work, feel free to copy and paste the following:

Ponnambalam S.G., Jawahar N. and Maheswaran. R. (2007). Hybrid Search Heuristics to Schedule Bottleneck Facility in Manufacturing Systems, Multiprocessor Scheduling, Theory and Applications, Eugene Levner (Ed.), ISBN: 978-3-902613-02-8, InTech, Available from:

http://www.intechopen.com/books/multiprocessor_scheduling_theory_and_applications/hybrid_search_heuristi cs_to_schedule_bottleneck_facility_in_manufacturing_systems

\section{INTECH}

open science | open minds

\author{
InTech Europe \\ University Campus STeP Ri \\ Slavka Krautzeka 83/A \\ 51000 Rijeka, Croatia \\ Phone: +385 (51) 770447 \\ Fax: +385 (51) 686166 \\ www.intechopen.com
}

\author{
InTech China \\ Unit 405, Office Block, Hotel Equatorial Shanghai \\ No.65, Yan An Road (West), Shanghai, 200040, China \\ 中国上海市延安西路65号上海国际贵都大饭店办公楼 405 单元 \\ Phone: +86-21-62489820 \\ Fax: +86-21-62489821
}


(C) 2007 The Author(s). Licensee IntechOpen. This chapter is distributed under the terms of the Creative Commons Attribution-NonCommercial-ShareAlike-3.0 License, which permits use, distribution and reproduction for non-commercial purposes, provided the original is properly cited and derivative works building on this content are distributed under the same license. 